\title{
The characteristic equation of the exceptional Jordan algebra: its eigenvalues, and their relation with mass ratios for quarks and leptons
}

\author{
Tejinder P. Singh \\ Tata Institute of Fundamental Research, Homi Bhabha Road, Mumbai 400005, India \\ e-mail: tpsingh@tifr.res.in
}

\begin{abstract}
We have recently proposed a pre-quantum, pre-space-time theory as a matrix-valued Lagrangian dynamics on an octonionic space-time. This pre-theory offers the prospect of unifying the internal symmetries of the standard model with gravity. It can also predict the values of free parameters of the standard model, because these parameters arising in the Lagrangian are related to the algebra of the octonions which define the underlying noncommutative space-time on which the dynamical degrees of freedom evolve. These free parameters are related to the algebra $J_{3}(\mathbb{O})$ [exceptional Jordan algebra] which in turn is related to the three fermion generations. The exceptional Jordan algebra [also known as the Albert algebra] is the finite dimensional algebra of 3x3 Hermitean matrices with octonionic entries. Its automorphism group is the exceptional Lie group $F_{4}$. These matrices admit a cubic characteristic equation whose eigenvalues are real and depend on the invariant trace, determinant, and an inner product made from the Jordan matrix. Also, there is some evidence in the literature that the group $F_{4}$ could play a role in the unification of the standard model symmetries, including the Lorentz symmetry. The octonion algebra is known to correctly yield the electric charge values $(0,1 / 3,2 / 3,1)$ for standard model fermions, via the eigenvalues of a $U(1)$ number operator, identified with $U(1)_{\mathrm{em}}$. In the present article, we use the same octonionic representation of the fermions to compute the eigenvalues of the characteristic equation of the Albert algebra, and compare the resulting eigenvalues with the known mass ratios for quarks and leptons. We find that the ratios of the eigenvalues correctly reproduce the [square root of the] known mass ratios for quarks and charged leptons. We also propose a diagrammatic representation of the standard model bosons, Higgs and three fermion generations, in terms of the octonions, exhibiting an $F_{4}$ and $E_{6}$ symmetry. In
\end{abstract}


conjunction with the trace dynamics Lagrangian, the Jordan eigenvalues also provide a first principles theoretical derivation of the low energy value of the fine structure constant, yielding the value 1/137.04006. The Karolyhazy correction to this value gives an exact match with the measured value of the constant, after assuming a specific value for the electro-weak symmetry breaking energy scale.

\section{CONTENTS}

I. Introduction

II. Three fermion generations, and physical eigenvalues from the characteristic equation of the Exceptional Jordan Algebra

III. An octonionic Lagrangian for the standard model

A. A Lagrangian on an 8D octonionic space-time

B. An octonionic diagrammatic representation for three fermion generations, and fourteen gauge bosons, and the Higgs

C. The Jordan eigenvalues and the low energy limiting value of the fine structure constant

IV. Discussion, and further developments: Jordan eigenvalues and mass-ratios

A. The Karolyhazy correction to the asymptotic value of fine structure constant 30

B. More Jordan eigenvalues for quarks and charged leptons

C. Update: Evidence of correlation between the Jordan eigenvalues and the mass ratios of quarks and charged leptons

D. Quantum non-locality

V. APPENDIX: Physical motivation for the present theory: quantum (field) theory without classical time, as a route to quantum gravity and unification

A. Why there must exist a formulation of quantum theory which does not refer to classical time? And why such a formulation must exist at all energy scales, not just at the Planck energy scale.

B. Why a quantum theory of gravity is needed at all energy scales, and not just at the Planck energy scale? And how that leads us to partially redefine what is 
meant by Planck scale: Replace Energy by Action.

C. What is Trace Dynamics? : Trace dynamics is quantisation, without imposing the Heisenberg algebra

D. Normed division algebras, trace dynamics, and relativity in higher dimensions. And how these relate to quantum field theory and the standard model

References

\section{INTRODUCTION}

We have recently proposed a pre-quantum, pre-space-time theory, which is a matrixvalued Lagrangian dynamics, written on an octonionic space-time. This theory generalises Adler's theory of trace dynamics [1-3], which is a pre-quantum theory on a four-dimensional Minkowski space-time $[4,5]$. It is a Lagrangian dynamics for Yang-Mills fields, fermions, and gravity. The algebra automorphisms of the octonions, which form the smallest exceptional Lie group $G_{2}$, play the role of unifying general coordinate transformations (i.e. space-time diffeomorphisms) with internal gauge transformations. We wrote down the Lagrangian for one generation of standard model fermions and gauge bosons, in this pre-theory. A Clifford algebra $C l(6, C)$ constructed from the octonion algebra is used to make spinors ['minimum left ideals' of $C l(6, C)]$ which represent the eight fermions of one generation, and their antiparticles, and their electro-color symmetry. Another $C l(6, C)$ made from the octonions describes the action of the Lorentz-weak symmetry on these octonions. These aspects of one-generation of fermions are confirmed by the Lagrangian dynamics constructed in the pre-theory. Our results are in agreement with the earlier work of Furey [6-8] and Stoica [9] for the Clifford algebra $C l(6, C)$ based description of one generation of standard model fermions. In our work, quantum field theory of the standard model emerges from the pretheory, at energies much lower than the Planck scale. The Appendix in Section V below summarises the theoretical background of the present article, as developed in our earlier papers $[4,10-12]$. The present paper should ideally be read as a continuation of [4]. We explain how the octonionic space-time, on which the fermions reside, fixes the dimensionless free parameters of the standard model [which appear in the octonionic Lagrangian] as a consequence of the properties of the algebra of the octonions, this being the exceptional 
Jordan algebra $J_{3}(8)$.

The possible connection between division algebras, exceptional Lie groups, and the standard model has been a subject of interest for many researchers in the last few decades [6-9, 13-32]. Our own interest in this connection stems from the following observation [4]. In the pre-geometric, pre-quantum theory of generalised trace dynamics, the definition of spin requires $4 \mathrm{D}$ space-time to be generalised to an $8 \mathrm{D}$ non-commutative space. In this case, an octonionic space is a possible, natural, choice for further investigation. We found that the additional four directions can serve as 'internal' directions and open a path towards a possible unification of the Lorentz symmetry with the standard model, with gravitation arising only as an emergent phenomenon. Instead of the Lorentz transformations and internal gauge transformations, the symmetries of the octonionic space are now described by the automorphisms of the octonion algebra. Remarkably enough, the symmetry groups of this algebra, namely the exceptional Lie groups, naturally have in them the desired symmetries [and only those symmetries, or higher ones built from them] of the standard model, including Lorentz symmetry, without the need for any fine tuning or adjustments. Thus the group of automorphisms of the octonions is $G_{2}$, the smallest of the five exceptional Lie groups $G_{2}, F_{4}, E_{6}, E_{7}, E_{8}$. The group $G_{2}$ has two intersecting maximal sub-groups [33], $S U(3) \times U(1)$ and $S U(2) \times S U(2)$, which between them account for the fourteen generators of $G_{2}$, and can possibly serve as the symmetry group for one generation of standard model fermions. The complexified Clifford algebra $C l(6, C)$ plays a very important role in establishing this connection. In particular, motivated by a map between the complexified octonion algebra and $C l(6, C)$, electric charge is defined as one-third the eigenvalue of a $U(1)$ number operator, which is identified with $U(1)_{e m}[6,8]$.

Describing the symmetries $S U(3) \times U(1)$ and $S U(2) \times S U(2)$ of the standard model [with Lorentz symmetry now included] requires two copies of the Clifford algebra $C l(6, C)$ whereas the octonion algebra yields only one such independent copy. It turns out that if boundary terms are not dropped from the Lagrangian of our theory, the Lagrangian describes three fermion generations [[4] and Section III below in the present paper], with the symmetry group now raised to $F_{4}$. This admits three intersecting copies of $G_{2}$, with the $S U(2) \times S U(2)$ in the intersection, and a Clifford algebra construction based on the three copies of the octonion algebra is now possible [34]. Attention thus shifts to investigating the connection between $F_{4}$ and the three generations of the standard model. 
$F_{4}$ is also the group of automorphisms of the exceptional Jordan algebra $[20,35,36]$. The elements of the algebra are 3x3 Hermitean matrices with octonionic entries. This algebra admits an important cubic characteristic equation with real eigenvalues. Now we know that the three fermion generations differ from each other only in the mass of the corresponding fermion, whereas the electric charge remains unchanged across the generations. This motivates us to ask: if the eigenvalues of the $U(1)$ number operator constructed from the octonion algebra represent electric charge, what is represented by the eigenvalues of the exceptional Jordan algebra? Could these eigenvalues bear a relation with mass ratios of quarks and leptons? This is the question investigated in the present paper and answered in the affirmative. Using the very same octonion algebra which was used to construct a state basis for standard model fermions, we calculate these eigenvalues. Remarkably, the eigenvalues are very simple to express, and bear a simple relation with electric charge. We describe how they relate to mass ratios. In particular we find that the ratios of the eigenvalues match with the square root of the mass ratios of charged fermions. [These eigenvalues are invariant under algebra automorphisms, the automorphism group being $F_{4}$, and the automorphisms of one chosen coordinate representation of the fermions, as below, give other equivalent coordinate representations for the same set of fermions. Octonions serve as coordinate systems on the eight dimensional octonionic space-time manifold on which the elementary fermions live. The Appendix at the end of this paper reviews this 8D space-time picture].

Thus we are asking that when the octonions representing the three fermion generations are used as the off-diagonal entries in the $3 \times 3$ Jordan matrices, and the diagonal entries are the electric charges, what is the physical interpretation of the eigenvalues of the characteristic equation of $J_{3}(\mathbb{O})$ ? These eigenvalues are made from the invariants of the algebra, and hence are themselves invariants. So they are likely to carry significant information about the standard model. This is what we explore in the present paper, and we argue that these eigenvalues inform us about mass-ratios of elementary particles, and about the coupling constants of the standard model.

Subsequently in the paper we propose a diagrammatic representation, based on octonions and $F_{4}$, of the fourteen gauge bosons, and the $(8 \times 2) \times 3=48$ fermions of three generations of standard model, along with the four Higgs. We attempt to explain why there are not three generations of bosons, and re-express our Lagrangian in a form which explicitly reflects this fact. We also argue as to how this Lagrangian might directly lead to the characteristic 
equation of the exceptional Jordan algebra, and reveal why the eigenvalues might be related to mass. Furthermore, we identify the standard model coupling constants in our Lagrangian, and by relating them to the eigenvalues of $J_{3}(\mathbb{O})$ we provide a theoretical derivation of the asymptotic fine structure constant value 1/137.xxx

It is known that since $F_{4}$ does not have complex representations, it cannot give a representation of the fermion states. It has hence been suggested that the correct representation could come from the next exceptional Lie group, $E_{6}$, which is the automorphism group of the complexified exceptional Jordan algebra. This aspect is currently being investigated by several researchers, including the present author. However, the standard model free parameters certainly cannot come from the characteristic equation related to $E_{6}$, because the roots of this equation are not real numbers in general. It is clear that the parameters must then come from the roots of the characteristic equation of $F_{4}$, which in a sense is the self-adjoint counterpart of the equation for $E_{6}$. It is in this spirit that the present investigation is carried out, and the results we find suggest that the present approach is indeed the correct one, as regards determining the model parameters. One must investigate $E_{6}$ for representations, but $F_{4}$ for the parameter values.

The plan of the paper is as follows. In the next section we recall the exceptional Jordan algebra, construct the octonionic representation of the three fermion generations, calculate the roots of the characteristic equation, and make some comments on mass-ratios and the roots. In Section III we construct the trace dynamics Lagrangian for three generations, along with the bosons, and we give a theoretical derivation of the asymptotic fine structure constant from first principles. In Section IV we calculate an additional set of eigenvalues for the fermions, generation wise; these provide evidence for violation of lepton universality. We then explain how the first set of Jordan eigenvalues in fact act as a definition of mass, quantised in units of Planck mass. We then show that mass ratios of charged fermions are obtained from these eigenvalues. In the Appendix in Section $\mathrm{V}$ we recall the motivation in earlier work, for developing this pre-theory, and we also include a few new insights. In particular we report on a 4D quaternionic version of the pre-theory, which describes the Lorentz-weak interaction of the leptons, based on an extension of the Lorentz algebra by $S U(2)$. In order to include quarks and the strong interaction, this $4 \mathrm{D}$ quaternionic pre-theory is extended to eight octonionic dimensions. 


\section{THREE FERMION GENERATIONS, AND PHYSICAL EIGENVALUES FROM THE CHARACTERISTIC EQUATION OF THE EXCEPTIONAL JORDAN ALGE- BRA}

The exceptional Jordan algebra $[\mathrm{EJA}] J_{3}(\mathbb{O})$ is the algebra of 3x3 Hermitean matrices with octonionic entries $[21,29,30,35]$

$$
X(\xi, x)=\left[\begin{array}{ccc}
\xi_{1} & x_{3} & x_{2}^{*} \\
x_{3}^{*} & \xi_{2} & x_{1} \\
x_{2} & x_{1}^{*} & \xi_{3}
\end{array}\right]
$$

It satisfies the characteristic equation $[21,29,30]$

$$
X^{3}-\operatorname{Tr}(X) X^{2}+S(X) X-\operatorname{Det}(X)=0 ; \quad \operatorname{Tr}(X)=\xi_{1}+\xi_{2}+\xi_{3}
$$

which is also satisfied by the eigenvalues $\lambda$ of this matrix

$$
\lambda^{3}-\operatorname{Tr}(X) \lambda^{2}+S(X) \lambda-\operatorname{Det}(X)=0
$$

Here the determinant is

$$
\operatorname{Det}(X)=\xi_{1} \xi_{2} \xi_{3}+2 \operatorname{Re}\left(x_{1} x_{2} x_{3}\right)-\sum_{1}^{3} \xi_{i} x_{i} x_{i}^{*}
$$

and $S(X)$ is given by

$$
S(X)=\xi_{1} \xi_{2}-x_{3} x_{3}^{*}+\xi_{2} \xi_{3}-x_{1} x_{1}^{*}+\xi_{1} \xi_{3}-x_{2}^{*} x_{2}
$$

The diagonal entries are real numbers and the off-diagonal entries are (real-valued) octonions. A star denotes an octonionic conjugate. The automorphism group of this algebra is the exceptional Lie group $F_{4}$. Because the Jordan matrix is Hermitean, it has real eigenvalues which can be obtained by solving the above-given eigenvalue equation.

In the present article we suggest that these eigenvalues carry information about mass ratios of quarks and leptons of the standard model, provided we suitably employ the octo- 
nionic entries and the diagonal real elements to describe quarks and leptons of the standard model. Building on earlier work $[6,7,9]$ we recently showed that the complexified Clifford algebra $C l(6, C)$ made from the octonions acting on themselves can be used to obtain an explicit octonionic representation for a single generation of eight quarks and leptons, and their anti-particles. In a specific basis, using the neutrino as the idempotent $V$, this representation is as follows $[4,6]$. The $\alpha$ are fermionic ladder operators of $C l(6, C)$ (please see Eqn. (34) of [4]).

$$
\begin{array}{rrr}
V=\frac{i}{2} e_{7} & {\left[V_{\nu} \text { Neutrino }\right]} \\
\alpha_{1}^{\dagger} V=\frac{1}{2}\left(e_{5}+i e_{4}\right) \times V=\frac{1}{4}\left(e_{5}+i e_{4}\right) \quad\left[\mathrm{V}_{\mathrm{ad} 1} \text { Anti }- \text { down quark }\right] \\
\alpha_{2}^{\dagger} V=\frac{1}{2}\left(e_{3}+i e_{1}\right) \times V=\frac{1}{4}\left(e_{3}+i e_{1}\right) \quad\left[\mathrm{V}_{\mathrm{ad} 2} \text { Anti }- \text { down quark }\right] \\
\alpha_{3}^{\dagger} V=\frac{1}{2}\left(e_{6}+i e_{2}\right) \times V=\frac{1}{4}\left(e_{6}+i e_{2}\right) \quad\left[\mathrm{V}_{\mathrm{ad} 3} \text { Anti }- \text { down quark }\right] \\
\alpha_{3}^{\dagger} \alpha_{2}^{\dagger} V=\frac{1}{4}\left(e_{4}+i e_{5}\right) & {\left[\mathrm{V}_{\mathrm{u} 1} \text { Up quark }\right]} \\
\alpha_{1}^{\dagger} \alpha_{3}^{\dagger} V==\frac{1}{4}\left(e_{1}+i e_{3}\right) & {\left[\mathrm{V}_{\mathrm{u} 2} \text { Up quark }\right]} \\
\alpha_{2}^{\dagger} \alpha_{1}^{\dagger} V=\frac{1}{4}\left(e_{2}+i e_{6}\right) & {\left[\mathrm{V}_{\mathrm{u} 3} \text { Up quark }\right]} \\
\alpha_{3}^{\dagger} \alpha_{2}^{\dagger} \alpha_{1}^{\dagger} V=-\frac{1}{4}\left(i+e_{7}\right) & {\left[\mathrm{V}_{\mathrm{e}+}\right. \text { Positron] }}
\end{array}
$$

The anti-particles are obtained from the above representation by complex conjugation [6].

Note: Eqn. (33) of [4] for the idempotent has an incorrectly written expression on the right hand side. Instead of $i e_{7} / 2$ as written there, the correct expression is $\left(1+i e_{7}\right) / 2$ [37]. Hence the idempotent $V$ in that paper should be $\left(1+i e_{7}\right) / 2$, not $i e_{7} / 2$. It has now been found however, that identification of the neutrino with the idempotent $V=\left(1+i e_{7}\right) / 2$ does not give the desired values for mass-ratios and coupling constants reported in the present paper [37]. We hence propose the Majorana particle interpretation for the neutrino, and identify the neutrino with $\left(V-V_{c c}\right) / 2$ where $V_{c c}$ is the complex conjugate of $V$. Hence the neutrino is $\left[\left(1+i e_{7}\right)-\left(1-i e_{7}\right)\right] / 4=i e_{7} / 2$, so that the octonionic representation of the neutrino remains the same as shown in [4] and is the one used in the present paper. Our results here seem to suggest that the neutrino is a Majorana particle, and not a Dirac particle.

Note: In Eqn. (34) of [4] the denominator in the expression for the positron should be 4, 
not 8. The correct expression for the positron is shown above in Eqn. (6).

In the context of the projective geometry of the octonionic projective plane $\mathbb{O} P^{2}$ it has been shown by Baez [23] that upto automorphisms, projections in EJA take one of the following four forms, having the respective invariant trace $0,1,2,3$.

$$
\begin{aligned}
& p_{0}=\left[\begin{array}{lll}
0 & 0 & 0 \\
0 & 0 & 0 \\
0 & 0 & 0
\end{array}\right] \\
& p_{1}=\left[\begin{array}{lll}
1 & 0 & 0 \\
0 & 0 & 0 \\
0 & 0 & 0
\end{array}\right] \\
& p_{2}=\left[\begin{array}{lll}
1 & 0 & 0 \\
0 & 1 & 0 \\
0 & 0 & 0
\end{array}\right] \\
& p_{3}=\left[\begin{array}{lll}
1 & 0 & 0 \\
0 & 1 & 0 \\
0 & 0 & 1
\end{array}\right]
\end{aligned}
$$

Since it has earlier been shown by Furey [6] that electric charge is defined in the division algebra framework as one-third of the eigenvalue of a $U(1)$ number operator made from the generators of the $S U(3)$ in $G_{2}$, we propose to identify the trace of the Jordan matrix with the sum of the charges of the three identically charged fermions across the three generations. Thus the trace zero Jordan matrix will have diagonal entries zero, and will represent the (neutrino, muon neutrino, tau-neutrino). The trace one Jordan matrix will have diagonal entries $(1 / 3,1 / 3,1 / 3)$ and will represent the (anti-down quark, anti-strange quark, anti-bottom quark). [Color is not relevant for determination of mass eigenvalues, and hence effectively we have four fermions per generation: two leptons and two quarks, after suppressing color]. The trace two Jordan matrix will have entries $(2 / 3,2 / 3,2 / 3)$ and will represent the (up quark, charm, top). Lastly, the trace three Jordan matrix will have entries $(1,1,1)$ and will represent (positron, anti-muon, anti-tau-lepton). 
We have thus identified the diagonal real entries of the four Jordan matrices whose eigenvalues we seek. We must next specify the octonionic entries in each of the four Jordan matrices. Note however that the above representation of the fermions of one generation is using complex octonions, whereas the entries in the Jordan matrices are real octonions. So we devise the following scheme for a one-to-one map from the complex octonion to a real octonion. Since we are ignoring color, we pick one out of the three up quarks, say $\left(e_{4}+i e_{5}\right)$, and one of three anti-down quarks, say $\left(e_{5}+i e_{4}\right)$. Since the representation for the electron and the neutrino use $e_{7}$ and a complex number, it follows that the four octonions we have picked form the quaternionic triplet $\left(e_{4}, e_{5}, e_{7}\right)$ [we use the Fano plane convention shown in the figure below]. Hence the four said octonions are in fact complex quaternions, thus belonging to the general form

$$
\left(a_{0}+i a_{1}\right)+\left(a_{2}+i a_{3}\right) e_{4}+\left(a_{4}+i a_{5}\right) e_{5}+\left(a_{6}+i a_{7}\right) e_{7}
$$

where the eight $a$-s are real numbers. By definition, we map this complex quaternion to the following real octonion:

$$
a_{0}+a_{1} e_{1}+a_{5} e_{2}+a_{3} e_{3}+a_{2} e_{4}+a_{4} e_{5}+a_{7} e_{6}+a_{6} e_{7}
$$

Note that the four real coefficients in the original complex quaternion have been kept in place, and their four imaginary counterparts have been moved to the octonion directions $\left(e_{1}, e_{2}, e_{3}, e_{6}\right)$ now as real numbers. Clearly, the map is reversible, given the real octonion we can construct the equivalent complex quaternion representing the fermion. We can now use this map and construct the following four real octonions for the neutrino, anti-down quark, up quark and the positron, respectively, after comparing with their complex octonion representation above.

$$
\begin{gathered}
V_{\nu}=\frac{i}{2} e_{7} \longrightarrow \frac{1}{2} e_{6} \\
V_{a d}=\frac{1}{4} e_{5}+\frac{i}{4} e_{4} \longrightarrow \frac{1}{4} e_{5}+\frac{1}{4} e_{3} \\
V_{u}=\frac{1}{4} e_{4}+\frac{i}{4} e_{5} \longrightarrow \frac{1}{4} e_{4}+\frac{1}{4} e_{2} \\
V_{e^{+}}=-\frac{i}{4}-\frac{1}{4} e_{7} \longrightarrow-\frac{1}{4} e_{1}-\frac{1}{4} e_{7}
\end{gathered}
$$




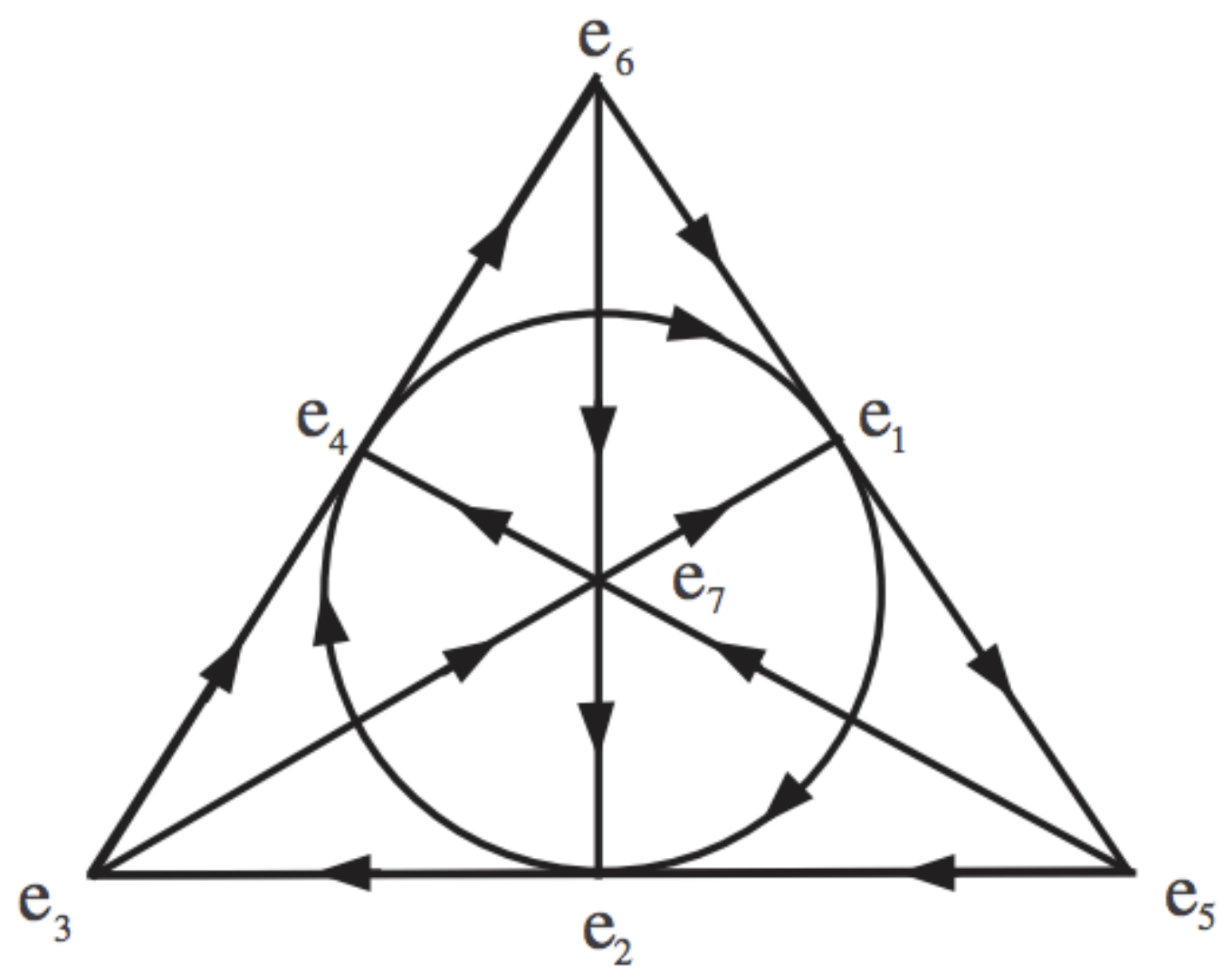

FIG. 1. The Fano plane.

These four real octonions will go, one each, in the four different Jordan matrices whose eigenvalues we wish to calculate. Next, we need the real octonionic representations for the four fermions [color suppressed] in the second generation and the four in the third generation. We propose to build these as follows, from the real octonion representations made just above for the first generation. Since $F_{4}$ has the inclusion $S U(3) \times S U(3)$, one $S U 3$ ) being for color and the other for generation, we propose to obtain the second generation by a $2 \pi / 3$ rotation on the first generation, and the third generation by a $2 \pi / 3$ rotation on the second generation. By this we mean the following construction, for the four respective Jordan matrices, as below. It is justified as follows: One of the two $S U(3)$ is color $S U(3)_{c}$ and has already been used up to write down the three different color states of each quark, with one pair of imaginary octonion directions fixed for a given color. The other $S U(3)$ is for generations. It is then 
evident from symmetry considerations that the corresponding higher generation quark of a given color can be obtained by $2 \pi / 3$ rotation on the first generation quark, while keeping the selected pair of octonionic directions fixed.

Up quark / Charm / Top: The up quark is $\left(e_{4} / 4+e_{2} / 4\right)$ We think of this as a 'plane' and rotate this octonion by $2 \pi / 3$ by left multiplying it by $e^{2 \pi e_{4} / 3}=-1 / 2+\sqrt{3} e_{4} / 2$. This will be the charm quark $V_{c}$. Then we left multiply the charm quark by $e^{2 \pi e_{4} / 3}$ to get the top quark $V_{t}$. Hence we have,

$V_{c}=\left(-1 / 2+\sqrt{3} e_{4} / 2\right) \times V_{u}=\left(-1 / 2+\sqrt{3} e_{4} / 2\right) \times\left(\frac{1}{4} e_{4}+\frac{1}{4} e_{2}\right)=-\frac{1}{8} e_{4}-\frac{1}{8} e_{2}-\frac{\sqrt{3}}{8}-\frac{\sqrt{3}}{8} e_{1}$

We have used the conventional multiplication rules for the octonions, which are reproduced

\begin{tabular}{|c|c|c|c|c|c|c|c|c|}
\hline & e0 & e1 & e2 & e4 & e3 & e5 & e6 & e7 \\
\hline e0 & 1 & e1 & e2 & e4 & e3 & e5 & e6 & e7 \\
\hline e1 & e1 & -1 & e4 & $-\mathrm{e} 2$ & e7 & e6 & -e5 & $-e 3$ \\
\hline e2 & e2 & $-\mathrm{e} 4$ & -1 & e1 & e5 & $-e 3$ & e7 & $-e 6$ \\
\hline e4 & e4 & e2 & $-e 1$ & -1 & -e6 & e7 & e3 & $-e 5$ \\
\hline e3 & e3 & -e7 & -e5 & e6 & -1 & e2 & -e4 & e1 \\
\hline e5 & e5 & -e6 & e3 & -e7 & $-\mathrm{e} 2$ & -1 & e1 & e4 \\
\hline e6 & e6 & e5 & $-e 7$ & -e3 & e4 & $-e 1$ & -1 & e2 \\
\hline e7 & e7 & e3 & e6 & e5 & -e1 & -e4 & -e2 & -1 \\
\hline
\end{tabular}

FIG. 2. The multiplication table for two octonions. Elements in the first column on the left, left multiply elements in the top row.

below in Fig. 2, for ready reference. Similarly, we can construct the top quark by a $2 \pi / 3$ 
rotation on the charm:

$$
\begin{aligned}
V_{t} & =\left(-1 / 2+\sqrt{3} e_{4} / 2\right) \times V_{c}=\left(-1 / 2+\sqrt{3} e_{4} / 2\right) \times\left(-\frac{1}{8} e_{4}-\frac{1}{8} e_{2}-\frac{\sqrt{3}}{8}-\frac{\sqrt{3}}{8} e_{1}\right) \\
& =-\frac{1}{8} e_{4}-\frac{1}{8} e_{2}+\frac{\sqrt{3}}{8}+\frac{\sqrt{3}}{8} e_{1}
\end{aligned}
$$

Next, we construct the anti-strange $V_{a s}$ and anti-bottom $V_{a b}$, by left-multiplication of the anti-down quark $V_{a d}$ by $e^{2 \pi e_{3} / 3}$.

$$
\begin{gathered}
V_{a s}=\left(-\frac{1}{2}+\frac{\sqrt{3}}{2} e_{3}\right) \times V_{a d}=\left(-\frac{1}{2}+\frac{\sqrt{3}}{2} e_{3}\right) \times\left(\frac{1}{4} e_{5}+\frac{1}{4} e_{3}\right) \\
=-\frac{1}{8} e_{5}-\frac{1}{8} e_{3}+\frac{\sqrt{3}}{8} e_{2}-\frac{\sqrt{3}}{8} \\
V_{a b}=\left(-\frac{1}{2}+\frac{\sqrt{3}}{2} e_{3}\right)\left(-\frac{1}{8} e_{5}-\frac{1}{8} e_{3}+\frac{\sqrt{3}}{8} e_{2}-\frac{\sqrt{3}}{8}\right) \\
=-\frac{1}{8} e_{5}-\frac{\sqrt{3}}{8} e_{2}-\frac{1}{8} e_{3}+\frac{\sqrt{3}}{8}
\end{gathered}
$$

Next, we construct the octonions for the anti-muon $V_{a \mu}$ and anti-tau-lepton $V_{a \tau}$ by left multiplying the positron $V_{e^{+}}$by $e^{2 \pi e_{1} / 3}$

$$
\begin{gathered}
V_{a \mu}=\left(-\frac{1}{2}+\frac{\sqrt{3}}{2} e_{1}\right) \times\left(-\frac{1}{4} e_{1}-\frac{1}{4} e_{7}\right) \\
=\frac{1}{8} e_{1}+\frac{1}{8} e_{7}+\frac{\sqrt{3}}{8}+\frac{\sqrt{3}}{8} e_{3} \\
V_{a \tau}=\left(-\frac{1}{2}+\frac{\sqrt{3}}{2} e_{1}\right) \times\left(\frac{1}{8} e_{1}+\frac{1}{8} e_{7}+\frac{\sqrt{3}}{8}+\frac{\sqrt{3}}{8} e_{3}\right) \\
=\frac{1}{8} e_{7}-\frac{\sqrt{3}}{8}+\frac{1}{8} e_{1}-\frac{\sqrt{3}}{8} e_{3}
\end{gathered}
$$

Lastly, we construct the octonions $V_{\nu \mu}$ for the muon neutrino and $V_{\nu \tau}$ for the tau neutrino, by left multiplying on the electron neutrino $V_{\nu}$ with $e^{2 \pi e_{6} / 3}$

$$
\left(-\frac{1}{2}+\frac{\sqrt{3}}{2} e_{6}\right) \times \frac{1}{2} e_{6}=-\frac{1}{4} e_{6}-\frac{\sqrt{3}}{4}
$$




$$
V_{\nu \tau}=\left(-\frac{1}{2}+\frac{\sqrt{3}}{2} e_{6}\right) \times\left(-\frac{1}{4} e_{6}-\frac{\sqrt{3}}{4}\right)=-\frac{1}{4} e_{6}+\frac{\sqrt{3}}{4}
$$

We now have all the information needed to write down the four Jordan matrices whose eigenvalues we will calculate. Diagonal entries are electric charge, and off-diagonal entries are octonions representing the particles. Using the above results we write down these four matrices explicitly. The neutrinos of three generations

$$
X_{\nu}=\left[\begin{array}{ccc}
0 & V_{\nu} & V_{\nu \mu}^{*} \\
V_{\nu}^{*} & 0 & V_{\nu \tau} \\
V_{\nu \mu} & V_{\nu \tau}^{*} & 0
\end{array}\right]
$$

The anti-down set of quarks of three generations [anti-down, anti-strange, anti-bottom]:

$$
X_{a d}=\left[\begin{array}{ccc}
\frac{1}{3} & V_{a d} & V_{a s}^{*} \\
V_{a d}^{*} & \frac{1}{3} & V_{a b} \\
V_{a s} & V_{a b}^{*} & \frac{1}{3}
\end{array}\right]
$$

The up set of quarks for three generations [up, charm, top]

$$
X_{u}=\left[\begin{array}{ccc}
\frac{2}{3} & V_{u} & V_{c}^{*} \\
V_{u}^{*} & \frac{2}{3} & V_{t} \\
V_{c} & V_{t}^{*} & \frac{2}{3}
\end{array}\right]
$$

The positively charged leptons of three generations [positron, anti-muon, anti-tau-lepton]

$$
X_{e+}=\left[\begin{array}{ccc}
1 & V_{e+} & V_{a \mu}^{*} \\
V_{e+}^{*} & 1 & V_{a \tau} \\
V_{a \mu} & V_{a \tau}^{*} & 1
\end{array}\right]
$$

Next, the eigenvalue equation corresponding to each of these Jordan matrices can be written down, after using the expressions given above for calculating the determinant and the function $S(X)$. Tedious but straightforward calculations with the octonion algebra give the following four cubic equations:

Neutrinos: We get $\operatorname{Tr}(X)=0, S(X)=-3 / 4, \operatorname{Det}(X)=0$, and hence the cubic equation 
and roots

$$
\lambda^{3}-\frac{3}{4} \lambda=0 \quad \text { ROOTS }:\left(-\sqrt{2} \sqrt{\frac{3}{8}}, 0, \sqrt{2} \sqrt{\frac{3}{8}}\right)
$$

Anti-down-quark + its higher generations [anti-down, anti-strange, anti-bottom]: We get $\operatorname{Tr}(X)=1, S(X)=-1 / 24, \operatorname{Det}(X)=-19 / 216$, and the following cubic equation and roots

$$
\begin{array}{r}
\lambda^{3}-\lambda^{2}-\frac{1}{24} \lambda+\frac{19}{216}=0 \\
\text { ROOTS }: \frac{1}{3}-\sqrt{\frac{3}{8}}, \frac{1}{3}, \frac{1}{3}+\sqrt{\frac{3}{8}}
\end{array}
$$

Up quark + its higher generations [up, charm, top]: We get $\operatorname{Tr}(X)=2, S(X)=23 / 24, \operatorname{Det}(X)=$ 5/108 and the following cubic equation and roots:

$$
\begin{array}{r}
\lambda^{3}-2 \lambda^{2}+\frac{23}{24} \lambda-\frac{5}{108}=0 \\
\text { ROOTS }: \frac{2}{3}-\sqrt{\frac{3}{8}}, \frac{2}{3}, \frac{2}{3}+\sqrt{\frac{3}{8}}
\end{array}
$$

Positron + its higher generations [positron, anti-muon, anti-tau-lepton]: We get $\operatorname{Tr}(X)=$ $3, S(X)=3-3 / 8, \operatorname{Det}(X)=1-3 / 8$ and the following cubic equation and roots:

$$
\begin{array}{r}
\lambda^{3}-3 \lambda^{2}+\left(3-\frac{3}{8}\right) \lambda-\left(1-\frac{3}{8}\right)=0 \\
\text { ROOTS : } 1-\sqrt{\frac{3}{8}}, 1,1+\sqrt{\frac{3}{8}}
\end{array}
$$

As expected from the known elementary properties of cubic equations, the sum of the roots is $\operatorname{Tr}(X)$, their product is $\operatorname{Det}(X)$, and the sum of their pairwise products is $S(X)$. Interestingly, this also shows that the sum of the roots is equal to the total electric charge of the three fermions under consideration in each of the respective cases. Whereas $S(X)$ and $\operatorname{Det}(X)$ are respectively related to an invariant inner product and an invariant trilinear form constructed from the Jordan matrix, their physical interpretation in terms of fermion properties remains to be understood.

The roots exhibit a remarkable pattern. In each of the four cases, one of the three roots is equal to the corresponding electric charge, and the other two roots are placed symmetrically on both sides of the middle root, which is the one equal to the electric charge. All three 
roots are positive in the up quark set and in the positron set, whereas the neutrino set and anti-down quark set have one negative root each, and the neutrino also has a zero root. It is easily verified that the calculation of eigenvalues for the anti-particles yields the same set of eigenvalues, upto a sign. In other words, the Jordan eigenvalue for the anti-particle is opposite in sign to that for the particle. The roots are summarised in the table below, and we see that they are composed of the electric charge, and the octonionic magnitude associated with the respective particle. [The octonionic magnitude $L_{P}^{2} / L^{2}$ is the sum $\sum x_{i} x^{i}$ over the three identically charged fermions of three generations, which appears in Equation (5) above.] One expects these roots to relate to masses of quarks and leptons

\section{The Jordan Eigenvalues}

\begin{tabular}{|c|c|c|c|}
\hline $\begin{array}{c}\text { Neutrinos: } \\
\text { Magntitude 3/4 }\end{array}$ & $-\frac{\sqrt{3}}{2}$ & $\mathbf{0}$ & \multicolumn{2}{|c|}{$\frac{\sqrt{3}}{2}$} \\
\hline $\begin{array}{c}\text { 1/3 Quarks: } \\
\text { Mag. 3/8 }\end{array}$ & $\frac{1}{3}-\sqrt{\frac{3}{8}}$ & $\frac{1}{3}$ & $\frac{1}{3}+\sqrt{\frac{3}{8}}$ \\
\hline $\begin{array}{c}\text { 2/3 Quarks } \\
\text { Mag. 3/8 }\end{array}$ & $\frac{2}{3}-\sqrt{\frac{3}{8}}$ & $\frac{2}{3}$ & $\frac{2}{3}+\sqrt{\frac{3}{8}}$ \\
\hline $\begin{array}{c}\text { Charged Leptons } \\
\text { Mag. 3/8 }\end{array}$ & $1-\sqrt{\frac{3}{8}}$ & $\mathbf{1}$ & $1+\sqrt{\frac{3}{8}}$ \\
\hline
\end{tabular}

These are all numbers in Base four !!

FIG. 3. The eigenvalues of the exceptional Jordan algebra for the various fermions. The eigenvalues are made from electric charge and the octonionic magnitude, and represent charge-mass of the corresponding fermion, in the pre-theory. The corresponding eigenmatrices [29] represent chargemass eigenstates. The $S U(3)_{c}$ and $U(1)$ constructed from the $C l(6)$ and the octonion algebra for one generation defines electric charge. However to define charge-mass and mass one must deal with $F_{4}$ and all three generations, not just one. 
for various reasons, and principally because the automorphism group of the complexified octonions contains the 4D Lorentz group as well, and the latter we know relates to gravity. Since mass is the source of gravity, we expect the Lorentz group to be involved in an essential way in any theory which predicts masses of elementary particles. And the group $F_{4}$, besides being related to $G_{2}$, and a possible candidate for the unification of the four interactions, is also the automorphism group of the EJA. We have motivated how the four projections of the EJA relate naturally to the four generation sets of the fermions. Thus there is a strong possibility that the eigenvalues of the characteristic equation of the EJA yield information about fermion mass ratios, especially it being a cubic equation with real roots. We make the following preliminary observations about the known mass ratios, and then provide a concrete analysis in Section IV.

The Jordan eigenvalues allow us to express the electric charge eigenstates of a fermion's three generations, as superpositions of mass eigenstates. That is why these eigenvalues determine mass ratios.

For the set (positron, anti-muon, anti-tau-lepton), the three respective masses are known to satisfy the following empirical relation, known as the Koide formula:

$$
\frac{m_{e}+m_{\mu}+m_{\tau}}{\left(\sqrt{m_{e}}+\sqrt{m_{\mu}}+\sqrt{m_{\tau}}\right)^{2}}=0.666661(7) \approx \frac{2}{3}
$$

For the three roots of the corresponding cubic equation (32) we get that

$$
2 \frac{\lambda_{1}^{2}+\lambda_{2}^{2}+\lambda_{3}^{2}}{\left(\lambda_{1}+\lambda_{2}+\lambda_{3}\right)^{2}}=2 \frac{[\operatorname{Tr}(X)]^{2}-2 S(X)}{[\operatorname{Tr}(X)]^{2}}=\frac{2}{3}\left(1+\frac{1}{4}\right) \approx 0.8333
$$

The factor $1 / 4$ comes from the sum of the absolute values of the three octonions which go into the related Jordan matrix. This observation suggests that the eigenvalues bear some relation with the square roots of the masses of the three charged leptons, though simply comparing square roots of their mass-ratios does not seem to yield any obvious relation with the eigenvalues. Further investigation is presented in Section IV. Here, we observe the following logarithmic ratios for masses of the charged leptons [taken as $0.5 \mathrm{MeV}, 105 \mathrm{MeV}$, 
$1777 \mathrm{Mev}$ ] and for the roots

$$
\begin{array}{ll}
\ln \left(\frac{105}{0.5}\right)^{1 / 2} \sim 2.67 ; & \frac{1}{1-\sqrt{\frac{3}{8}}} \sim 2.58 \\
\ln \left(\frac{1777}{0.5}\right)^{1 / 2} \sim 4.09 ; \quad \frac{1+\sqrt{\frac{3}{8}}}{1-\sqrt{\frac{3}{8}}} \sim 4.16 \\
\ln \left(\frac{1777}{105}\right)^{1 / 2} \sim 1.41 ; \quad \frac{1+\sqrt{\frac{3}{8}}}{1} \sim 1.61
\end{array}
$$

For the up quark set though, we see a correlation in terms of square roots of masses.

In the case of the up quark set, the following approximate match is observed between the ratios of the eigenvalues, and the mass square root ratios of the masses of up, charm and top quark. For the sake of this estimate we take these three quark masses to be [2.3, 1275, 173210] in Mev [38]. The following ratios are observed:

$$
\begin{gathered}
\sqrt{\frac{1275}{2.3}} \sim 23.55 ; \quad \frac{\frac{2}{3}+\sqrt{\frac{3}{8}}}{\frac{2}{3}-\sqrt{\frac{3}{8}}} \approx 23.56 \\
\sqrt{\frac{173210}{1275}} \sim 11.66 ; \quad \frac{\frac{2}{3}}{\frac{2}{3}-\sqrt{\frac{3}{8}}} \approx 12.28 \\
\sqrt{\frac{173210}{2.3}} \sim 274.42 ; \quad\left(\frac{\frac{2}{3}+\sqrt{\frac{3}{8}}}{\frac{2}{3}-\sqrt{\frac{3}{8}}}\right) \times\left(\frac{\frac{2}{3}}{\frac{2}{3}-\sqrt{\frac{3}{8}}}\right) \approx 289.23
\end{gathered}
$$

Within the error bars on the masses of the up set of quarks, the two sets of ratios are seen to agree with each other upto second decimal place.

Considering that one of the roots is negative in the anti-down-quark set, we cannot directly relate the eigenvalues to mass ratios. The same is true for the neutrino set, where one root is negative and one root is zero. In section IV we propose that the correct quantity to examine is the square-root of mass (in dimensionless units), which can take both positive sign and negative sign: $\pm \sqrt{m}$. The Jordan eigenvalues relate to the square-root of either sign, with the eigenvalue for anti-particle being opposite in sign to that for the particle. 
The case of the neutrino is especially instructive, and shows how non-zero mass could arise fundamentally, even when the electric charge is zero. In this case, the non-zero contribution comes from the inner product related quantity $S(X)$, and therein from the absolute magnitude of the octonions in the Jordan matrix, which necessarily has to be non-zero. We thus see that masses are derivative concepts, obtained from the three more fundamental entities, namely the electric charge, and the geometric invariants $S(X)$ and $\operatorname{Det}(X)$, with the last two necessarily being defined commonly for the three generations. And since mass is the source of gravity, this picture is consistent with gravity and space-time geometry being emergent from the underlying geometry of the octonionic space which algebraically determines the properties of the elementary particles. We note that there are no free parameters in the above analysis, no dimensional quantities, and no assumption has been put by hand. Except that we identify the octonions with elementary fermions. The numbers which come out from the above analysis are number-theoretic properties of the octonion algebra.

These observations suggest a possible fundamental relation between eigenvalues of the EJA and particle masses. In the next section, we provide further evidence for such a connection, based on our proposal for unification based on division algebras and a matrix-valued Lagrangian dynamics.

\section{AN OCTONIONIC LAGRANGIAN FOR THE STANDARD MODEL}

\section{A. A Lagrangian on an $8 \mathrm{D}$ octonionic space-time}

The action and Lagrangian for the three generations of standard model fermions, fourteen gauge bosons, and four potential Higgs bosons, are given by [4]

$$
\frac{S}{C_{0}}=\int d \tau \mathcal{L} \quad ; \quad \mathcal{L}=\frac{1}{2} \operatorname{Tr}\left[\frac{L_{p}^{2}}{L^{2}} \dot{\widetilde{Q}}_{1}^{\dagger} \dot{\widetilde{Q}}_{2}\right]
$$

Here,

$$
\dot{\widetilde{Q}}_{1}^{\dagger}=\dot{\widetilde{Q}}_{B}^{\dagger}+\frac{L_{p}^{2}}{L^{2}} \beta_{1} \dot{\widetilde{Q}}_{F}^{\dagger} ; \quad \dot{\widetilde{Q}}_{2}=\dot{\widetilde{Q}}_{B}+\frac{L_{p}^{2}}{L^{2}} \beta_{2} \dot{\widetilde{Q}}_{F}
$$

and

$$
\dot{\widetilde{Q}}_{B}=\frac{1}{L}\left(i \alpha q_{B}+L \dot{q}_{B}\right) ; \quad \dot{\widetilde{Q}}_{F}=\frac{1}{L}\left(i \alpha q_{F}+L \dot{q}_{F}\right)=
$$


By defining

$$
q_{1}^{\dagger}=q_{B}^{\dagger}+\frac{L_{P}^{2}}{L^{2}} \beta_{1} q_{F}^{\dagger} \quad ; \quad q_{2}=q_{B}+\frac{L_{P}^{2}}{L^{2}} \beta_{2} q_{F}
$$

we can express the Lagrangian as

$$
\begin{aligned}
\mathcal{L} & =\frac{L_{P}^{2}}{2 L^{2}} \operatorname{Tr}\left[\left(\dot{q}_{1}^{\dagger}+\frac{i \alpha}{L} q_{1}^{\dagger}\right) \times\left(\dot{q}_{2}+\frac{i \alpha}{L} q_{2}\right)\right] \\
& =\frac{L_{P}^{2}}{2 L^{2}} \operatorname{Tr}\left[\dot{q}_{1}^{\dagger} \dot{q}_{2}-\frac{\alpha^{2}}{L^{2}} q_{1}^{\dagger} q_{2}+\frac{i \alpha}{L} q_{1}^{\dagger} \dot{q}_{2}+\frac{i \alpha}{L} \dot{q}_{1}^{\dagger} q_{2}\right]
\end{aligned}
$$

We now expand each of these four terms inside of the trace Lagrangian, using the definitions of $q_{1}$ and $q_{2}$ given above:

$$
\begin{aligned}
& \dot{q}_{1}^{\dagger} \dot{q}_{2}=\dot{q}_{B}^{\dagger} \dot{q}_{B}+\frac{L_{P}^{2}}{L^{2}} \dot{q}_{B}^{\dagger} \beta_{2} \dot{q}_{F}+\frac{L_{P}^{2}}{L^{2}} \beta_{1} \dot{q}_{F}^{\dagger} \dot{q}_{B}+\frac{L_{P}^{4}}{L^{4}} \beta_{1} \dot{q}_{F}^{\dagger} \beta_{2} \dot{q}_{F} \\
& q_{1}^{\dagger} q_{2}=q_{B}^{\dagger} q_{B}+\frac{L_{P}^{2}}{L^{2}} q_{B}^{\dagger} \beta_{2} q_{F}+\frac{L_{P}^{2}}{L^{2}} \beta_{1} q_{F}^{\dagger} q_{B}+\frac{L_{P}^{4}}{L^{4}} \beta_{1} q_{F}^{\dagger} \beta_{2} q_{F} \\
& q_{1}^{\dagger} \dot{q}_{2}=q_{B}^{\dagger} \dot{q}_{B}+\frac{L_{P}^{2}}{L^{2}} q_{B}^{\dagger} \beta_{2} \dot{q}_{F}+\frac{L_{P}^{2}}{L^{2}} \beta_{1} q_{F}^{\dagger} \dot{q}_{B}+\frac{L_{P}^{4}}{L^{4}} \beta_{1} q_{F}^{\dagger} \beta_{2} \dot{q}_{F} \\
& \dot{q}_{1}^{\dagger} q_{2}=\dot{q}_{B}^{\dagger} q_{B}+\frac{L_{P}^{2}}{L^{2}} \dot{q}_{B}^{\dagger} \beta_{2} q_{F}+\frac{L_{P}^{2}}{L^{2}} \beta_{1} \dot{q}_{F}^{\dagger} q_{B}+\frac{L_{P}^{4}}{L^{4}} \beta_{1} \dot{q}_{F}^{\dagger} \beta_{2} q_{F}
\end{aligned}
$$

In our recent work, we suggested this Lagrangian, having the symmetry group $F_{4}$, as a candidate for unification. There are fourteen gauge bosons (equal to the number of generators of $G_{2}$ ). These are the eight gluons, the three weak isospin vector bosons, the photon, and the two Lorentz bosons. These bosons, along with one Higgs, can be accounted for by the four bosonic terms which form the first column in the above four sub-equations. The remaining twelve terms were proposed to describe three fermion generations and three Higgs, with the three generations being motivated by the triality of $S O(8)$. However, one important question which has not been addressed there is: why does triality not give rise to three copies of the bosons?! In the framework of the present approach we tentatively explore the following answer. We know that the even-grade Grassmann numbers which form the entries of the bosonic matrices are made from even-number products of odd-grade (fermionic) Grassmann numbers, and the latter are in a sense more basic. Could it then be that bosonic degrees of freedom are made from fermionic degrees of freedom? If this were to be so, it could prevent the tripling of bosons, if we think of them as arising at the 'intersections' of the octonionic directions which represent fermions. 


\section{B. An octonionic diagrammatic representation for three fermion generations, and} fourteen gauge bosons, and the Higgs

The seven imaginary unit octonions are used to make the Fano plane, which has seven points and seven lines [adding to fourteen elements; points and lines have equal status]. If we include the real direction [we have assumed $\dot{q}_{B 0}$ to be self-adjoint] also, we get an equivalent of a 3-D cube where the eight vertices now stand for the eight octonions, with one of them [the 'origin'] standing for the real line. As explained by Baez: "The Fano plane is the projective plane over the 2-element field $Z_{2}$. In other words, it consists of lines through the origin in the vector space $Z_{2}^{3}$. Since every such line contains a single nonzero element, we can also think of the Fano plane as consisting of the seven nonzero elements of $Z_{2}^{3}$. If we think of the origin in $Z_{2}^{3}$ as corresponding to 1 in $\mathbb{O}$, we get the following picture of the octonions". This picture is Fig. 3 below, borrowed from Baez [23]. Considering points, lines and faces together, this structure has 26 elements $[8+12+6=26]$. Motivated by this representation of the octonion, and the triality of $S O(8)$, we propose the following diagrammatic representation of the standard model fermions, gauge bosons, and Higgs as shown in Fig. 4. It motivates us to think of bosons as arising as 'intersections' of the elements representing fermions. We have taken four copies of the Baez cube, with the central one at the intersection of the other three, and used them to represent the elementary particles. We now attempt to describe Fig. 4 in some detail. There is a central black-colored cube (henceforth a cube is an octonion) in the front, which represents the fourteen gauge bosons and the four Higgs bosons; we will return to this cube shortly. Then there are three more (colored) cubes: one to the left, one at the back, and one at the bottom. These are marked as Gen I, Gen II and Gen III, and represent the three fermion generations. Let us focus first on the octonion on the left, which is Gen I, and where the eight vertices have been marked $\left(e_{0}, e_{1}, e_{2}, e_{3}, e_{4}, e_{5}, e_{6}, e_{7}\right)$ just as in the Baez cube. If $e_{0}$ were to be excluded, this cube becomes the Fano plane [Fig. 1 above] and the arrows marked in the Gen. I cube follow the same directions as in the Fano plane. In this Gen I cube, leaving out all those elements which are at the intersection with the central bosonic cube, and leaving out the face on the far left, we are left with sixteen elements: four points, eight lines, and four faces. The four points are shown in blue and are $\left(e_{3}, e_{5}, e_{6}, e_{7}\right)$. The eight lines are: $\left(e_{4} e_{3}, e_{7} e_{2}, e_{3} e_{7}, e_{7} e_{6}, e_{5} e_{6}, e_{6} e_{4}, e_{5} e_{0}, e_{6} e_{1}\right)$. The four planes are: $\left(e_{4} e_{3} e_{7} e_{2}\right),\left(e_{0} e_{5} e_{6} e_{1}\right),\left(e_{7} e_{2} e_{1} e_{6}\right),\left(e_{3} e_{4} e_{0} e_{5}\right)$. Between them, these sixteen 


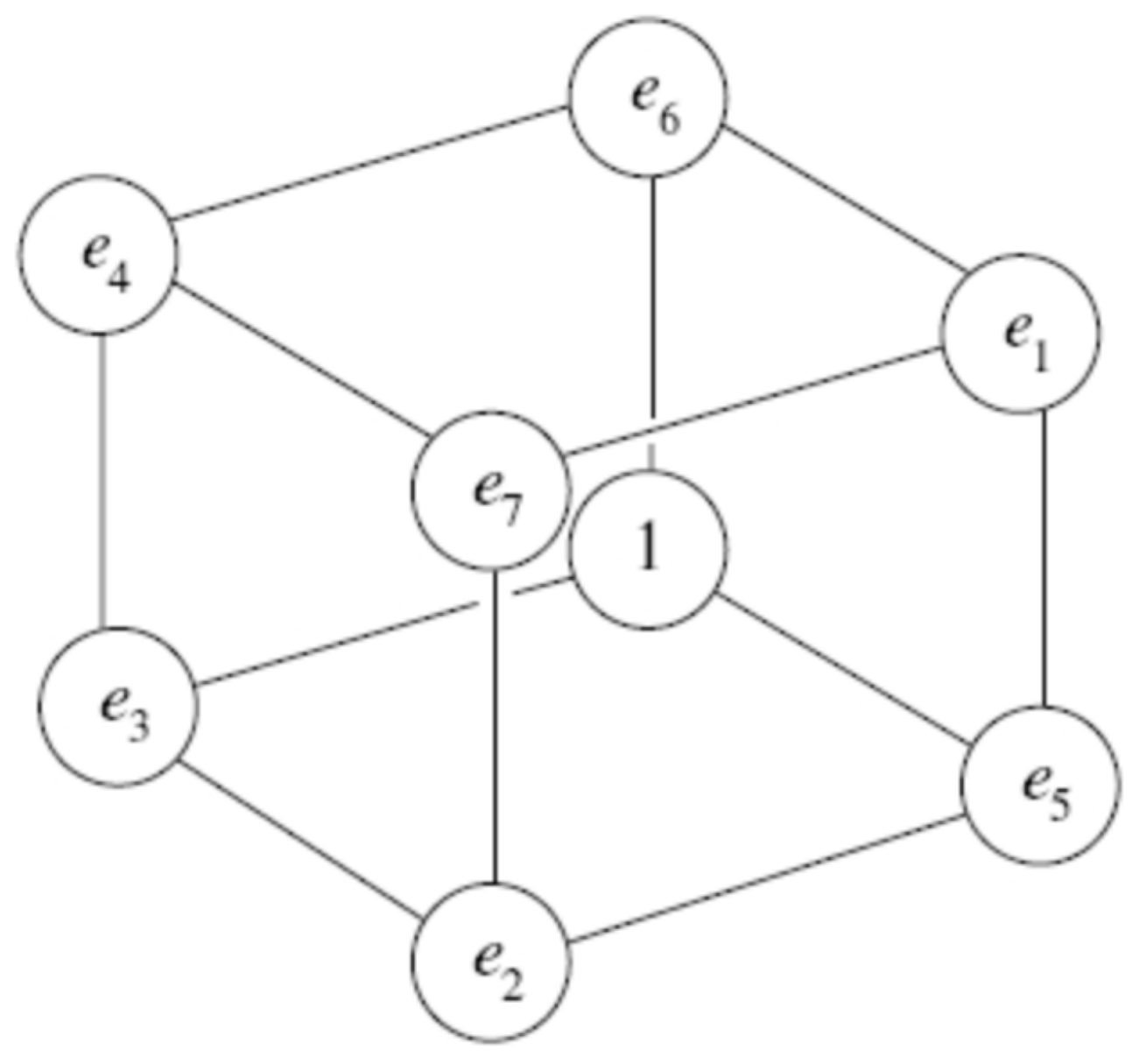

FIG. 4. The octonions [From Baez [23]].

elements represent the eight fermions and their anti-particles in one generation, one particle / anti-particle per octonionic element.

The up quark, the down quark, and their anti-particles of one particular color are (marked by) the four lines $\left(e_{4} e_{3}, e_{7} e_{2}, e_{0} e_{5}, e_{6} e_{1}\right)$. The points $\left(e_{3}, e_{5}, e_{6}, e_{7}\right)$ mark $u, d$ of a second color, and the lines $\left(e_{3} e_{7}, e_{7} e_{6}, e_{3} e_{5}, e_{5} e_{6}\right)$ mark the $u, d$ of the third color. The four planes mark the electron, the neutrino, and their anti-particles. Between them, these sixteen elements have an $S U(3)$ symmetry: they can be correlated to the $(8+8) \mathrm{D}$ particle basis constructed by Furey, from the $S U(3)$ in $G_{2}$. Next, the Gen II and Gen III along with Gen I has another $S U(3)$ symmetry, which is responsible for the three generations. These three fermionic cubes represent three intersecting copies of $G_{2}$ each cube having an $S U(3)$ symmetry. The three-way intersection is $S U(2) X S U(2)$, this being the black central cube, and the bosons 


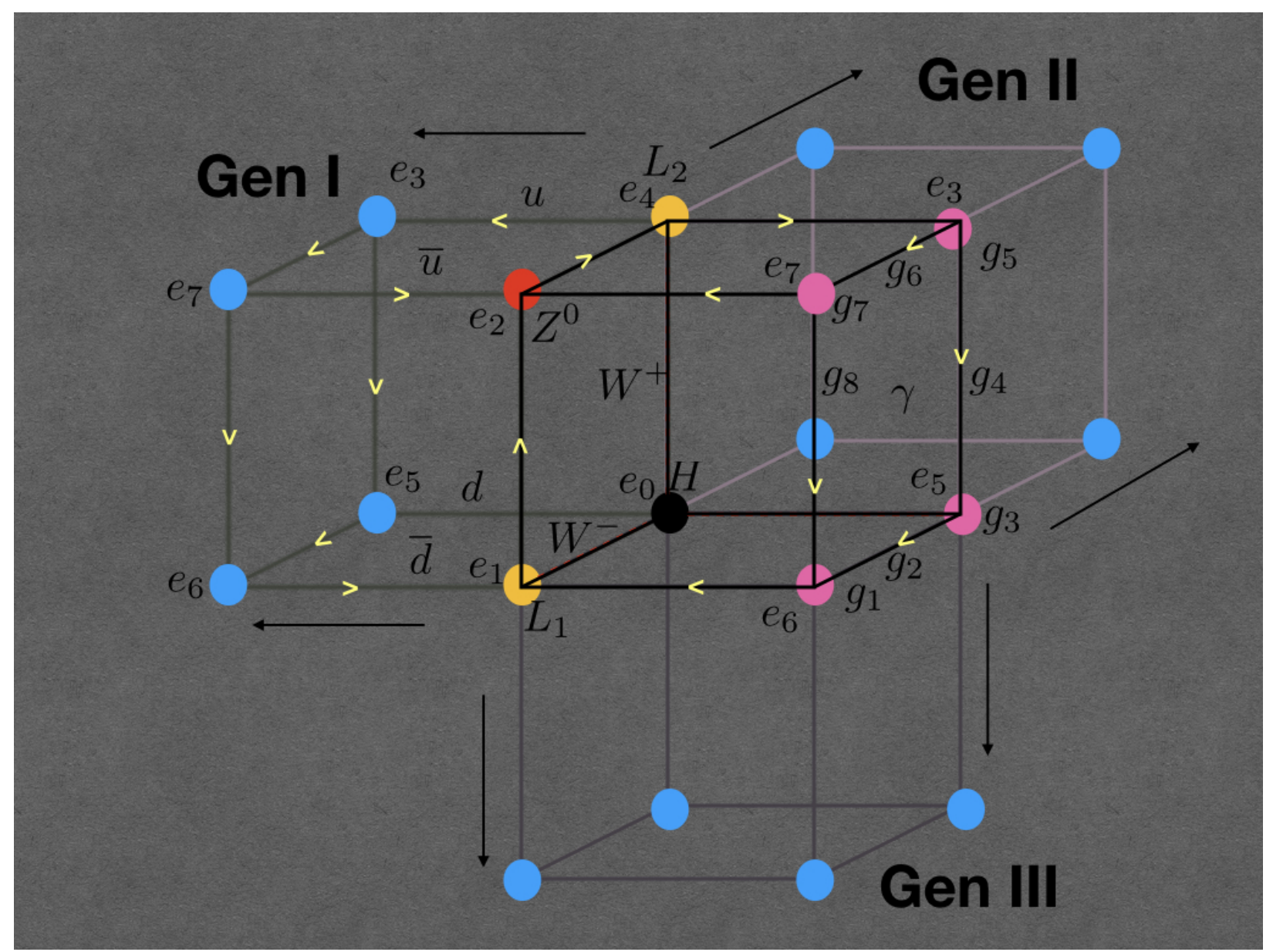

FIG. 5. The elementary particles of the standard model with three generations, represented through octonions in an $F_{4}$ diagram.. Please see text for a detailed explanation.

lie on this cube. At the same time the fermionic cubes make contact with the bosonic cube, enabling the bosons to act on the fermions.

We now try to understand the central bosonic cube. First we count the number of its elements: it gets a total of $3 \times 10=30$ elements from the three side cubes, which when added to its own 26 elements gives a total of 56. But there are a lot of common elements, so that the actual number of independent elements is much smaller, and we enumerate them now. Three points are shared two-way and three points shared three-way and the point $e_{0}$ is shared four-way; that reduces the count to 44. Nine lines are shared: three of them three way, and six of them two way, reducing the count to 32. The shared three planes reduce the count to 29. We now account for the assignment of bosons to these 29 locations.

The eight gluons are on the front right, marked by the pink points, and lines labelled $g_{1}$ to $g_{8}$, and the photon is assigned to the plane $\left(e_{3} e_{7} e_{6} e_{5}\right)$ on the front right enclosed by the 
gluons. The two Lorentz bosons are the yellow points $e_{4}$ and $e_{1}$ also marked $L_{2}$ and $L_{1}$. The three vector bosons are marked by the lines $e_{0} e_{1}, e_{0} e_{4}$ and the point $e_{2}$, also marked $Z^{0}$. The Higgs $H$ is at the four way real point $e_{0}$. Three more Higgs are shown as follows: two planes per Higgs, e.g. the plane $e_{0} e_{4} e_{2} e_{1}$ and the mirror fermionic plane $e_{3} e_{5} e_{6} e_{7}$ on the far left in Gen I. Analogously, another Higgs is given by the bosonic plane $e_{0} e_{1} e_{6} e_{5}$ and its mirror fermionic plane at the front bottom in Gen III. The third Higgs is given by the bosonic plane $e_{0} e_{4} e_{3} e_{5}$ and its mirror fermionic plane at the back in Gen II. This way 21 elements are used up. The remaining 8 un-used elements (six lines and two planes) are assigned to eight terms in the Lagrangian representing the action of the spacetime symmetry on the gluons: these are the terms $\dot{q}_{B} q_{B}^{\dagger}$ and $\dot{q}_{B}^{\dagger} q_{B}$ in (46).

The bosonic cube lies in the intersection of the three $G_{2}$ and hence does not triplicate during the $S U(3)$ rotation which generates the three fermion generations. The symmetry group of the theory is the 52 dimensional group $F_{4}$, with $8 \times 3=24$ generators coming from the three fermionic cubes, and the rest 28 from the bosonic sector $[14+2 \times 3+8=28]$. This diagram does suggest that one could investigate bosonic degrees of freedom as made from pairs of fermion degrees of freedom. With this tentative motivation, we return to our Lagrangian, and seek to write it explicitly as for a single generation of bosons, and three generations of fermions. Upon examination of the sub-equations in Eqn. (46) we find that the last column has terms bilinear in the fermions, and we would like to make it appear just as the second and third column do, so that we can explicitly have three fermion generations. With this intent, we propose the following assumed definitions of the bosonic degrees of freedom, by recasting the four terms in the last column of Eqn. (46):

$$
\begin{aligned}
& \frac{L_{P}^{4}}{L^{4}} \beta_{1} \dot{q}_{F}^{\dagger} \beta_{2} \dot{q}_{F} \equiv \frac{L_{P}^{2}}{L^{2}} \dot{q}_{B} \beta_{2} \dot{q}_{F}+\frac{\alpha^{2}}{L^{2}} A \\
& \frac{L_{P}^{4}}{L^{4}} \beta_{1} q_{F}^{\dagger} \beta_{2} q_{F} \equiv \frac{L_{P}^{2}}{L^{2}} q_{B} \beta_{2} q_{F}+A \\
& \frac{L_{P}^{4}}{L^{4}} \beta_{1} q_{F}^{\dagger} \beta_{2} \dot{q}_{F} \equiv \frac{L_{P}^{2}}{L^{2}} q_{B}^{\dagger} \beta_{1} \dot{q}_{F}^{\dagger}+B \\
& \frac{L_{P}^{4}}{L^{4}} \beta_{1} \dot{q}_{F}^{\dagger} \beta_{2} q_{F} \equiv \frac{L_{P}^{2}}{L^{2}} \dot{q}_{B}^{\dagger} \beta_{1} q_{F}^{\dagger}-B
\end{aligned}
$$

where $A$ and $B$ are bosonic matrices which drop out on summing the various terms to get the full Lagrangian, With this redefinition, the sub-equations Eqn. (46) can be now written 
in the following form after rewriting the last column:

$$
\begin{aligned}
& \dot{q}_{1}^{\dagger} \dot{q}_{2}=\dot{q}_{B}^{\dagger} \dot{q}_{B}+\frac{L_{P}^{2}}{L^{2}} \dot{q}_{B}^{\dagger} \beta_{2} \dot{q}_{F}+\frac{L_{P}^{2}}{L^{2}} \beta_{1} \dot{q}_{F}^{\dagger} \dot{q}_{B}+\frac{L_{P}^{2}}{L^{2}} \dot{q}_{B} \beta_{2} \dot{q}_{F} \\
& q_{1}^{\dagger} q_{2}=q_{B}^{\dagger} q_{B}+\frac{L_{P}^{2}}{L^{2}} q_{B}^{\dagger} \beta_{2} q_{F}+\frac{L_{P}^{2}}{L^{2}} \beta_{1} q_{F}^{\dagger} q_{B}+\frac{L_{P}^{2}}{L^{2}} q_{B} \beta_{2} q_{F} \\
& q_{1}^{\dagger} \dot{q}_{2}=q_{B}^{\dagger} \dot{q}_{B}+\frac{L_{P}^{2}}{L^{2}} q_{B}^{\dagger} \beta_{2} \dot{q}_{F}+\frac{L_{P}^{2}}{L^{2}} \beta_{1} q_{F}^{\dagger} \dot{q}_{B}+\frac{L_{P}^{2}}{L^{2}} q_{B}^{\dagger} \beta_{1} \dot{q}_{F}^{\dagger} \\
& \dot{q}_{1}^{\dagger} q_{2}=\dot{q}_{B}^{\dagger} q_{B}+\frac{L_{P}^{2}}{L^{2}} \dot{q}_{B}^{\dagger} \beta_{2} q_{F}+\frac{L_{P}^{2}}{L^{2}} \beta_{1} \dot{q}_{F}^{\dagger} q_{B}+\frac{L_{P}^{2}}{L^{2}} \dot{q}_{B}^{\dagger} \beta_{1} q_{F}^{\dagger}
\end{aligned}
$$

The terms now look harmonious and we can see a structure emerging - the first column are bosonic terms and these are not triples. The remaining terms are four sets of three each [to which their adjoints will eventually get added] which can clearly describe three generations of the four sets, which is what we had in the Jordan matrices in the previous section. Putting it all together, we can now rewrite the Lagrangian so that it explicitly looks like the one for gauge bosons and four sets of three generations of fermions, as in the Jordan matrix:

$$
\begin{gathered}
\mathcal{L}=\frac{L_{P}^{2}}{2 L^{2}} \operatorname{Tr}\left[\left(\dot{q}_{1}^{\dagger}+\frac{i \alpha}{L} q_{1}^{\dagger}\right) \times\left(\dot{q}_{2}+\frac{i \alpha}{L} q_{2}\right)\right] \\
=\frac{L_{P}^{2}}{2 L^{2}} \operatorname{Tr}\left[\dot{q}_{1}^{\dagger} \dot{q}_{2}-\frac{\alpha^{2}}{L^{2}} q_{1}^{\dagger} q_{2}+\frac{i \alpha}{L} q_{1}^{\dagger} \dot{q}_{2}+\frac{i \alpha}{L} \dot{q}_{1}^{\dagger} q_{2}\right] \\
\equiv \frac{L_{P}^{2}}{2 L^{2}} \operatorname{Tr}\left[\mathcal{L}_{\text {bosons }}+\mathcal{L}_{\text {set } 1}+\mathcal{L}_{\text {set } 2}+\mathcal{L}_{\text {set } 3}+\mathcal{L}_{\text {set } 4}\right]
\end{gathered}
$$

where

$$
\begin{gathered}
\mathcal{L}_{\text {bosons }}=\dot{q}_{B}^{\dagger} \dot{q}_{B}-\frac{\alpha^{2}}{L^{2}} q_{B}^{\dagger} q_{B}+\frac{i \alpha}{L} q_{B}^{\dagger} \dot{q}_{B}+\frac{i \alpha}{L} \dot{q}_{B}^{\dagger} q_{B} \\
\mathcal{L}_{\text {set } 1}=\frac{L_{P}^{2}}{L^{2}} \dot{q}_{B}^{\dagger} \beta_{2} \dot{q}_{F}+\frac{L_{P}^{2}}{L^{2}} \beta_{1} \dot{q}_{F}^{\dagger} \dot{q}_{B}+\frac{L_{P}^{2}}{L^{2}} \dot{q}_{B} \beta_{2} \dot{q}_{F} \\
\mathcal{L}_{\text {set } 2}=-\frac{\alpha^{2}}{L^{2}}\left(\frac{L_{P}^{2}}{L^{2}} q_{B}^{\dagger} \beta_{2} q_{F}+\frac{L_{P}^{2}}{L^{2}} \beta_{1} q_{F}^{\dagger} q_{B}+\frac{L_{P}^{2}}{L^{2}} q_{B} \beta_{2} q_{F}\right) \\
\mathcal{L}_{\text {set } 3}=\frac{i \alpha}{L}\left(\frac{L_{P}^{2}}{L^{2}} q_{B}^{\dagger} \beta_{2} \dot{q}_{F}+\frac{L_{P}^{2}}{L^{2}} \beta_{1} q_{F}^{\dagger} \dot{q}_{B}+\frac{L_{P}^{2}}{L^{2}} q_{B}^{\dagger} \beta_{1} \dot{q}_{F}^{\dagger}\right) \\
\mathcal{L}_{\text {set } 4}=\frac{i \alpha}{L}\left(\frac{L_{P}^{2}}{L^{2}} \dot{q}_{B}^{\dagger} \beta_{2} q_{F}+\frac{L_{P}^{2}}{L^{2}} \beta_{1} \dot{q}_{F}^{\dagger} q_{B}+\frac{L_{P}^{2}}{L^{2}} \dot{q}_{B}^{\dagger} \beta_{1} q_{F}^{\dagger}\right)
\end{gathered}
$$

We see that each of these four fermionic sets could possibly be related to a Jordan matrix, after including the adjoint part. We also see that different coupling constants appear in 
different sets with identical coupling in third and fourth set and no coupling in the first set. The first set could possibly describe neutrinos, charged leptons and quarks (gravitational and weak interaction), the second set charged leptons and quarks, and the third and fourth set the quarks. To establish this explicitly, equations of motion remain to be worked out and then related to the eigenvalue problem. As noted earlier, $L$ relates to mass, and this approach could reveal how the eigenvalues of the EJA characteristic equation relate to mass. This investigation is currently in progress, and proceeds along the following lines. We take the self-adjoint part of the above Lagrangian, because that part is the one which leads to quantum field theory in the emergent approximation after coarse-graining the underlying theory. [The anti-self-adjoint part is negligible in the approximation in which quantum field theory emerges, and when it becomes significant, spontaneous localisation occurs, and classical space-time and the macroscopic universe emerges]. We vary the self-adjoint part of the Lagrangian with respect to the bosonic degree of freedom, and with respect to the three 8D-fermionic degrees of freedom, representing the three fermion generations. This yields four equations of motion, three of which are coupled matrix-valued Dirac equations for the three generations. These three coupled equations are solved by a state vector which is a threevector made of three 8-spinors. The eigenvalue problem for three coupled matrix equations is likely solved by the exceptional Jordan algebra, the algebra of 3x3 Hermitean matrices with octonionic entries, where the diagonal entries are identified with electric charge. That the diagonal entries are electric charge is justified by the form of the Lagrangian above, especially as written in Eqn. (45), because we see $\alpha / L$ as the coefficient of the potential, and its square appearing in the electrodynamics term (52) in this latest form of the Lagrangian above. This coefficient in front of the terms in Eqn. (52) then gets identified with the fine structure constant, as below.

The symmetry group associated with the self-adjoint part of the Lagrangian is $F_{4}$. The symmetry group associated with the full Lagrangian, including the anti-self-adjoint part, could possibly be $E_{6}$ - this is under investigation. 


\section{The Jordan eigenvalues and the low energy limiting value of the fine structure} constant

If we examine the Lagrangian term for the charged leptons in Eqn. (52), the dimensionless coupiing constant $C$ in front of it is (upto a sign):

$$
C \equiv \alpha^{2} \frac{L_{P}^{4}}{L^{4}}
$$

[The operator terms of the form $q_{B} q_{F}$ etc. in (52) have been correspondingly made dimensionless by dividing by $\left.L_{P}^{2}\right]$. We assume that $\ln \alpha$ is linearly proportional to the electric charge, and that the proportionality constant is the Jordan eigenvalue corresponding to the anti-down quark. The electric charge $1 / 3$ of the anti-down quark seems to be the right choice for determining $\alpha$, it being the smallest non-zero value [and hence possibly the fundamental value] of the electric charge, and also because the constant $\alpha$ appears as the coupling in front of the supposed quark terms in the Lagrangian, as in Eqns. (53) and (54). We hence define $\alpha$ by

$$
\ln \alpha \equiv \lambda_{a d} q_{a d}=\left[\frac{1}{3}-\sqrt{\frac{3}{8}}\right] \times \frac{1}{3} \quad \Longrightarrow \quad \alpha^{2} \approx=0.83025195149
$$

where $\lambda_{a d}$ is the Jordan eigenvalue corresponding to the anti-down quark, as given by Eqn. (30) and $q_{a d}$ is the electric charge of the anti-down quark $(=1 / 3)$. In order to arrive at this relation for $\alpha$, we asked in what way $\alpha$ could vary with $q$, if it was allowed to vary? We then made the assumption that $d \alpha / d q \propto \alpha$. In the resulting linear dependence of $\ln \alpha$ on $q$, we froze the value of $\alpha$ at that given by the smallest non-zero charge value $1 / 3$, taking the proportionality constant to be the corresponding Jordan eigenvalue. This dependence also justifies that had we fixed $\alpha$ from the zero charge of the neutrino, $\alpha$ would have been one, as it in fact is, in our Lagrangian. We are investigating if this way of constructing $\alpha$ can be further justified from the Lagrangian dynamics.

As for the value of $L_{P} / L$, we identify it with one-half of that part of the Jordan eigenvalue which modifies the contribution coming from the electric charge. [For an explanation of the origin of the factor of one-half, see the next paragraph]. Thus from the eigenvalues found above, we deduce that for neutrinos, quarks and charged leptons, the quantity $L_{P}^{2} / L^{2}$ 
takes the respective values $(3 / 16,3 / 32,3 / 32)$. These values are equal to one-fourth of the respective octonionic magnitudes. Thus the coupling constant $C$ defined above can now be calculated, with $\alpha^{2}$ as given above, and $L_{P}^{2} / L^{2}=3 / 32$. Furthermore, since the electric charge $q$, the way it is conventionally defined, has dimensions such that $q^{2}$ has dimensions (Energy $\times$ Length), we measure $q^{2}$ in Planck units $E_{P l} \times L_{P}=\hbar c$. We hence define the fine structure constant by $C=\alpha^{2} L_{P}^{4} / L^{4} \equiv e^{2} / \hbar c$, where $e$ is the electric charge of electron / muon / tau-lepton in conventional units. We hence get the value of the fine structure constant to be

$$
C=\alpha^{2} L_{P}^{4} / L^{4} \equiv e^{2} / \hbar c=\exp \left[\left[\frac{1}{3}-\sqrt{\frac{3}{8}}\right] \times \frac{2}{3}\right] \times \frac{9}{1024} \approx 0.00729713=\frac{1}{137.04006}
$$

The CODATA 2018 value of the fine structure constant is

$$
0.0072973525693(11)=1 / 137.035999084(21)
$$

Our calculated value differs from the measured value in the seventh decimal place. In the next section, we show how incorporating the Karolyhazy length correction gives an exact match with the CODATA 2018 value, if we assume a specific value for the electro-weak symmetry breaking energy scale.

Why did we identify $L_{P} / L$ with one-half of the octonionic magnitude $\sqrt{3 / 8}$ rather than with the magnitude $\sqrt{3 / 8}$ itself? The answer lies in the physical interpretation originally assigned to the length scale L. [Please see the discussion below Eqn. (69) of [10]]. The length $L$ for an object of mass $m$ is interpreted as the Schwarzschild radius $2 \mathrm{Gm} / \mathrm{c}^{2}$ of an object of mass $m$, so that $L_{P} / L=L_{P} c^{2} / 2 G m$, which is one-half the Compton wave-length (in Planck units) and not the Compton wavelength itself. Assuming that the octonionic magnitude has to be identified with Compton wavelength (in units of Planck length), it hence has to be divided by one-half, before equating it to $L_{P} / L$. This justifies taking $L_{P}^{2} / L^{2}=1 / 4 \times 3 / 8=3 / 32$.

Once a theoretical derivation of the asymptotic fine structure constant is known, one can write the electric charge $e$ as

$$
e=(3 / 32) \exp [1 / 9-1 / \sqrt{24}]\left(\hbar L_{P} / t_{P}\right)^{1 / 2}
$$


where $L_{P}$ and $t_{P}$ are Planck length and Planck time respectively - obviously their ratio is the speed of light. In our theory, there are only three fundamental dimensionful quantities: Planck length, Planck time, and a constant with dimensions of action, which in the emergent quantum theory is identified with Planck's constant $\hbar$. We now see that electric charge is not independent of these three fundamental dimensionful constants. It follows from them. Planck mass is also constructed from these three, and electron mass will be expressed in terms of Planck mass, if only we could understand why the electron is some $10^{22}$ times lighter than Planck mass. Such a small number cannot come from the octonion algebra. In all likelihood, the cosmological expansion up until the electroweak symmetry breaking is playing a role here.

Thus electric charge and mass can both be expressed in terms of Planck's constant, Planck length and Planck time. This encourages us to think of electromagnetism, as well the other internal symmetries, entirely in geometric terms. This geometry is dictated by the $F_{4}$ symmetry of the exceptional Jordan algebra.

\section{DISCUSSION, AND FURTHER DEVELOPMENTS: JORDAN EIGENVAL- UES AND MASS-RATIOS}

We have not addressed the question as to how these discrete order one eigenvalues might relate to actual low values of fermion masses, which are much lower than Planck mass. We speculatively suggest the following scenario, which needs to be explored further. The universe is eight-dimensional, not four. The other four internal dimensions are not compactified; rather the universe is very 'thin' in those dimensions but they are expanding as well. There are reasons having to do with the so-called Karolyhazy uncertainty relation [39], because of which the universe expands in the internal dimensions at one-third the rate, on the logarithmic scale, compared to our $3 \mathrm{D}$ space. That is, if the $4 \mathrm{D}$ scale factor is $a(\tau)$, the internal scale factor is $a_{\text {int }}^{1 / 3}(\tau)$, in Planck length units. Taking the size of the observed universe to be about $10^{61}$ Planck units, the internal dimensions have a width approximately $10^{20}$ Planck units, which is about $10^{-13} \mathrm{~cm}$, thus being in the quantum domain. Classical systems have an internal dimension width much smaller than Planck length, and hence they effectively stay in [and appear to live in] four dimensional space-time. Quantum systems probe all eight dimensions, and hence live in an octonionic universe. 
The universe began in a unified phase, via an inflationary 8D expansion possibly resulting as the aftermath of a huge spontaneous localisation event in a 'sea of atoms of space-timematter' [10]. The mass values are set, presumably in Planck scale, at order one values dictated by the eigenvalues reported in the present paper. Cosmic inflation scales down these mass values at the rate $a^{1 / 3}(\tau)$, where $a(\tau)$ is the $4 \mathrm{D}$ expansion rate. Inflation ends after about sixty e-folds, because seeding of classical structures breaks the color-elctro-weakLorentz symmetry, and classical spacetime emerges as a broken Lorentz symmetry. The electro-weak symmetry breaking is actually a electro-weakLorentz symmetry breaking, which is responsible for the emergence of gravity, weak interaction being its short distance limit. There is no reheating after inflation; rather inflation resets the Planck scale in the vicinity of the electro-weak scale, and the observed low fermion mass values result. The electroweak symmetry breaking is mediated by the Lorentz symmetry, in a manner consistent with the conventional Higgs mechanism. It is not clear why inflation should end specifically at the electro-weak scale: this is likely dictated by when spontaneous localisation becomes significant enough for classical spacetime to emerge. It is a competition between the strength of the electro-colour interaction which attempts to bind the fermions, and the inflationary expansion which opposes this binding. Eventually, the expanding universe cools enough for spontaneous localisation to win, so that the Lorentz symmetry is broken. It remains to prove from first principles that this happens at around the electro-weak scale and also to investigate the possibly important role that Planck mass primordial black holes might play in the emergence of classical spacetime. I would like to thank Roberto Onofrio for correspondence which has influenced these ideas. See also [40].

\section{A. The Karolyhazy correction to the asymptotic value of fine structure constant}

In accordance with the Karolyhazy uncertainty relation (Eqn. (9) of [39]) a measured length $l$ has a 'quantum gravitational' correction $\Delta l$ given by

$$
(\Delta l)^{3}=L_{P}^{2} l
$$

For the purpose of the present discussion we shall assume an equality sign here, i.e. that the numerical constant of proportionality between the two sides of the equation is unity. And, 
for the sake of the present application to the fine structure constant, we rewrite this relation as

$$
\delta \equiv \frac{L_{P}}{\Delta l}=\left(\frac{L_{P}}{l}\right)^{1 / 3}
$$

We set $l \equiv l_{f}$ where $l_{f}$ is the length scale $\left(\approx 10^{-16} \mathrm{~cm}\right)$ associated with electro-weak symmetry breaking, where classical space-time emerges from the prespacetime, prequantum theory. The assumption being that when the universe evolves from the Planck scale to the electro-weak scale [while remaining in the unbroken symmetry phase], the inverse of the octonionic length associated with the charged leptons (this being $\sqrt{3 / 32}$ ) is reset, because of the Karolyhazy correction, to

$$
\sqrt{\frac{3}{32}} \longrightarrow \sqrt{\frac{3}{32}}+\delta_{f} \equiv \sqrt{\frac{3}{32}}+\left(\frac{L_{P}}{l_{f}}\right)^{1 / 3}
$$

We can also infer this corrected length as the four-dimensional space-time measure of the length, which differs from the eight dimensional octonionic value $\sqrt{3 / 32}$ by the amount $\delta_{f}$. If we take $l_{f}$ to be $10^{-16} \mathrm{~cm}$, the correction $\delta_{f}$ is of the order $2 \times 10^{-6}$. The correction to the asymptotic value (57) of the fine structure constant is then

$$
C=\alpha^{2} L_{P}^{4} / L^{4} \equiv e^{2} / \hbar c=\alpha^{2}\left[\sqrt{\frac{3}{32}}+\left(\frac{L_{P}}{l_{f}}\right)^{1 / 3}\right]^{4}
$$

For $l_{f}=10^{-16} \mathrm{~cm}=198 \mathrm{GeV}^{-1}$, we get the corrected value of the fine structure constant to be 0.00729737649, which overshoots the measured CODATA 2018 value at the eighth decimal place. The electroweak scale is generally assumed to lie in the range $100-1000 \mathrm{GeV}$. The value $l_{f}=1.3699526 \times 10^{-16} \mathrm{~cm}=144.530543605 \mathrm{GeV}^{-1}$ reproduces the CODATA 2018 value 0.0072973525693 of the asymptotic fine structure constant. The choice $l_{f}^{-1}=246 \mathrm{GeV}$ gives the value 0.00729739452 , whereas the choice $l_{f}^{-1}=159.5 \pm 1.5 \mathrm{GeV}$ gives the range (0.00729736049, 0.00729735908). $100 \mathrm{GeV}$ gives the value 0.00729732757 which is smaller than the measured value. $1000 \mathrm{GeV}$ gives 0.00729754842 . Thus in the entire $100-1000$ $\mathrm{GeV}$ range, the derived constant agrees with the measured value at least to the sixth decimal place, which is reassuring. The purpose of the present exercise is to show that the Karolyhazy correction leads to a correction to the asymptotic value of the fine structure constant which is in the desired range - a striking fact by itself. In principle, our theory should predict the 
precise value of the electroweak symmetry breaking scale. Since that analysis has not yet been carried out, we predict that the ColorElectro-WeakLorentz symmetry breaking scale is 144.something $\mathrm{GeV}$, because only then the theoretically calculated value of the asymptotic fine structure constant matches the experimentally measured value.

The above discussion of the asymptotic low energy value of the fine structure constant should not be confused with the running of the constant with energy. Once we recover classical spacetime and quantum field theory from our theory, after the ColorElectro-WeakLorentz symmetry breaking, conventional RG arguments apply, and the running of couplings with energy is to be worked out as is done conventionally. Such an analysis of running couplings will however be valid only up until the broken symmnetry is restored - it is not applicable in the prespacetime prequantum phase. In this sense, our theory is different from GUTs. Once there is unification, Lorentz symmetry is unified with internal symmetries - the exact energy scale at which that happens remains to be worked out.

How then does the Planck scale prespacetime, prequantum theory know about the low energy asymptotic value of the fine structure constant? The answer to this question lies in the Lagrangian given in (49) and in particular the Lagrangian term (52) for the charged leptons. In determining the asymptotic fine structure constant from here, we have neglected the modification to the coupling that will come from the presence of $q_{B}$ and $q_{F}$. This is analogous to examining the asymptotic, flat spacetime limit of a spacetime geometry due to a source - gravity is evident close to the source, but hardly so, far from it. Similarly, there is a Minkowski-flat analog of the octonionic space, wherein the effect of $q_{B}$ and $q_{F}$ (which in effect 'curve' the octonionic space) is ignorable, and the asymptotic fine structure can be computed. The significance of the non-commutative, non-associative octonion algebra and the Jordan eigenvalues lies in that they already determine the coupling constants, including their asymptotic values. This is a property of the algebra, even though the interpretation of a particular constant as the fine structure constant comes from the dynamics, i.e. the Lagrangian, as it should, on physical grounds.

On a related note about this approach to unification, we recall that the symmetry group in our theory is $U(1) \times S U(3) \times S U(2) \times S U(2)$. This bears resemblance to the study of a left-right symmetric extension of the standard model by Boyle [41] in the context of the complexified exceptional Jordan algebra. This $L-R$ model has exceptional phenomenological 
promise, and it appears that the unbroken phase [prior to the ColorElectro-WeakLorentz symmetry breaking] of the L-R model is well-described by our Lagrangian (49) for three generations. This gives further justification for exploring the phenomenology of this Lagrangian.

\section{B. More Jordan eigenvalues for quarks and charged leptons}

Assuming that the mechanism for mass generation of neutrinos is different from that for the electrically charged fermions, we can set aside the neutrinos for the time being, and calculate additional new eigenvalues of the exceptional Jordan algebra in yet another way. We club the three charged fermions of the first generation to make a $3 \times 3$ Jordan matrix, with the octonionic entries assigned as: $x_{1}$ is the anti-down quark, $x_{2}$ is the up quark, and $x_{3}$ is the positron. Analogously, the octonionic entries for the second generation are such that $x_{1}$ is the anti-strange quark, $x_{2}$ is the charm quark, and $x_{3}$ is the anti-muon. For the third generation Jordan matrix, $x_{1}$ is the anti-bottom quark, $x_{2}$ is the top quark, and $x_{3}$ is the anti-tau-lepton. For each of the three Jordan matrices, the diagonal entries are the electric charges i.e. $(1 / 3,2 / 3,1)$, so that the trace is 2 for each of the three Jordan matrices. $S(X)$ is also the same for each generation, and is equal 61/72. The determinant is different in each of the three cases and is given by

$\operatorname{Det}(G e n I)=-\frac{25}{576}-\frac{9}{576} ; \quad \operatorname{Det}(G e n I I)=-\frac{25}{576}+\frac{\sqrt{3}}{576} ; \quad \operatorname{Det}(G e n I I I)=-\frac{25}{576}-\frac{\sqrt{3}}{576}$

The three Jordan matrices for which we are now calculating the eigenvalues are hence given as follows, one for each generation of two quarks and one charged lepton:

$$
\begin{aligned}
\text { GenI : } & {\left[\begin{array}{ccc}
1 & V_{e+} & V_{u p}^{*} \\
V_{e+}^{*} & 2 / 3 & V_{a d} \\
V_{u p} & V_{a d}^{*} & 1 / 3
\end{array}\right] } \\
\text { GenII : } & {\left[\begin{array}{ccc}
1 & V_{a \mu} & V_{c}^{*} \\
V_{a \mu}^{*} & 2 / 3 & V_{a s} \\
V_{c} & V_{a s}^{*} & 1 / 3
\end{array}\right] }
\end{aligned}
$$




$$
\text { GenIII : } \quad\left[\begin{array}{ccc}
1 & V_{a \tau} & V_{t}^{*} \\
V_{a \tau}^{*} & 2 / 3 & V_{a b} \\
V_{t} & V_{a b}^{*} & 1 / 3
\end{array}\right]
$$

The notation and octonionic representation is the same as earlier in the paper. For each of the three generations the eigenvalues are given by the following set of three real roots, each of which is positive (hence a total of nine unequal roots):

$$
\begin{array}{r}
\lambda_{1}=\frac{2}{3}+2 \sqrt{-Q} \cos \left(\frac{\theta}{3}\right) \\
\lambda_{2}=\frac{2}{3}+2 \sqrt{-Q} \cos \left(\frac{\theta+2 \pi}{3}\right) \\
\lambda_{3}=\frac{2}{3}+2 \sqrt{-Q} \cos \left(\frac{\theta+4 \pi}{3}\right)
\end{array}
$$

Here, the angle $\theta$ is defined by

$$
\theta \equiv \cos ^{-1}\left(\frac{R}{\sqrt{-Q^{3}}}\right)
$$

and the function $Q$ is the same for each of the three generations:

$$
Q=\frac{3 S(X)-\operatorname{Tr}^{2}(X)}{9}=-\frac{35}{216}
$$

whereas the function $R$ differs slightly amongst the three generations because the determinant is different for each of them:

$$
R=-\frac{1}{6} \operatorname{Tr}(X) S(X)+\frac{1}{27} \operatorname{Tr}^{3}(X)+\frac{1}{2} \operatorname{Det}(X)=-\frac{1}{3} \times \frac{61}{72}+\frac{8}{27}+\frac{1}{2} \operatorname{Det}(X)
$$

The angle $\theta$ in the case of the three generations can thus be calculated, and is given in radians by

$$
\theta_{I}=1.81270 ; \quad \theta_{I I}=1.69730 ; \quad \theta_{I I I}=1.74837
$$


The roots can now be computed and have the following set of three values each, for each of the three generations, respectively:

$$
\begin{array}{r}
\lambda_{1 I}=2 / 3-0.70985=-0.04318 ; \quad \lambda_{2 I}=2 / 3+0.02599=0.69266 ; \quad \lambda_{3 I}=2 / 3+0.68385=1.35052 \\
\lambda_{1 I I}=2 / 3-0.71565=-0.04898 ; \quad \lambda_{2 I I}=2 / 3+0.03844=0.70511 ; \quad \lambda_{3 I I}=2 / 3+.6731=1.34387 \\
\lambda_{1 I I I}=2 / 3-0.72738=-0.06071 ; \quad \lambda_{2 I I I}=2 / 3+0.06484=0.73151 ; \quad \lambda_{3 I I I}=2 / 3+0.66252=1.32919
\end{array}
$$

As is evident, for every generation, the roots are shifted around the middle electric charge value of $2 / 3$, as if undergoing a rotation determined by $\theta$, with one root coming out larger than $2 / 3$, and the other two roots smaller than $2 / 3$.

In combination with the nine eigenvalues found earlier in the paper for the six quarks and three charged leptons, we now have a total of 18 unequal roots, only one of which is negative. The nine roots found earlier could be labeled as 'horizontal' roots, calculated across three generations in three sets, one set each for the three fermions with identical nonzero electric charge. The nine roots found now could labeled as 'vertical' roots, calculated per generation, using the three fermions with non-zero charge. The only negative root is the horizontal root for the anti-down quark. The full set of 18 roots are shown in the table below [Figure 5], two per charged fermion. In each of the nine cells of the table, the upper entry is a horizontal root, and the lower entry is a vertical root. Using the up quark as a benchmark, eight ratios can be defined from the nine vertical roots, and another eight ratios from the nine horizontal roots. The fact that the angles $\theta_{I}, \theta_{I I}$ and $\theta_{I I I}$ are different for the three generations suggests a possible violation of lepton universality. As far as mass ratios are concerned, it turns out that the horizontal eigenvalues are the only ones to be used, and the vertical ones are not used at all in calculating mass ratios.

\section{Update: Evidence of correlation between the Jordan eigenvalues and the mass ratios of quarks and charged leptons}

In the first generation, we note the positron mass to be $0.511 \mathrm{Mev}$, the up quark mass to be $2.3 \pm 0.7 \pm 0.5 \mathrm{MeV}$, and the down quark mass to be $4.8 \pm 0.5 \pm 0.3 \mathrm{MeV}$. The uncertainties in the two quark masses permit us to make the following proposal: the square- 


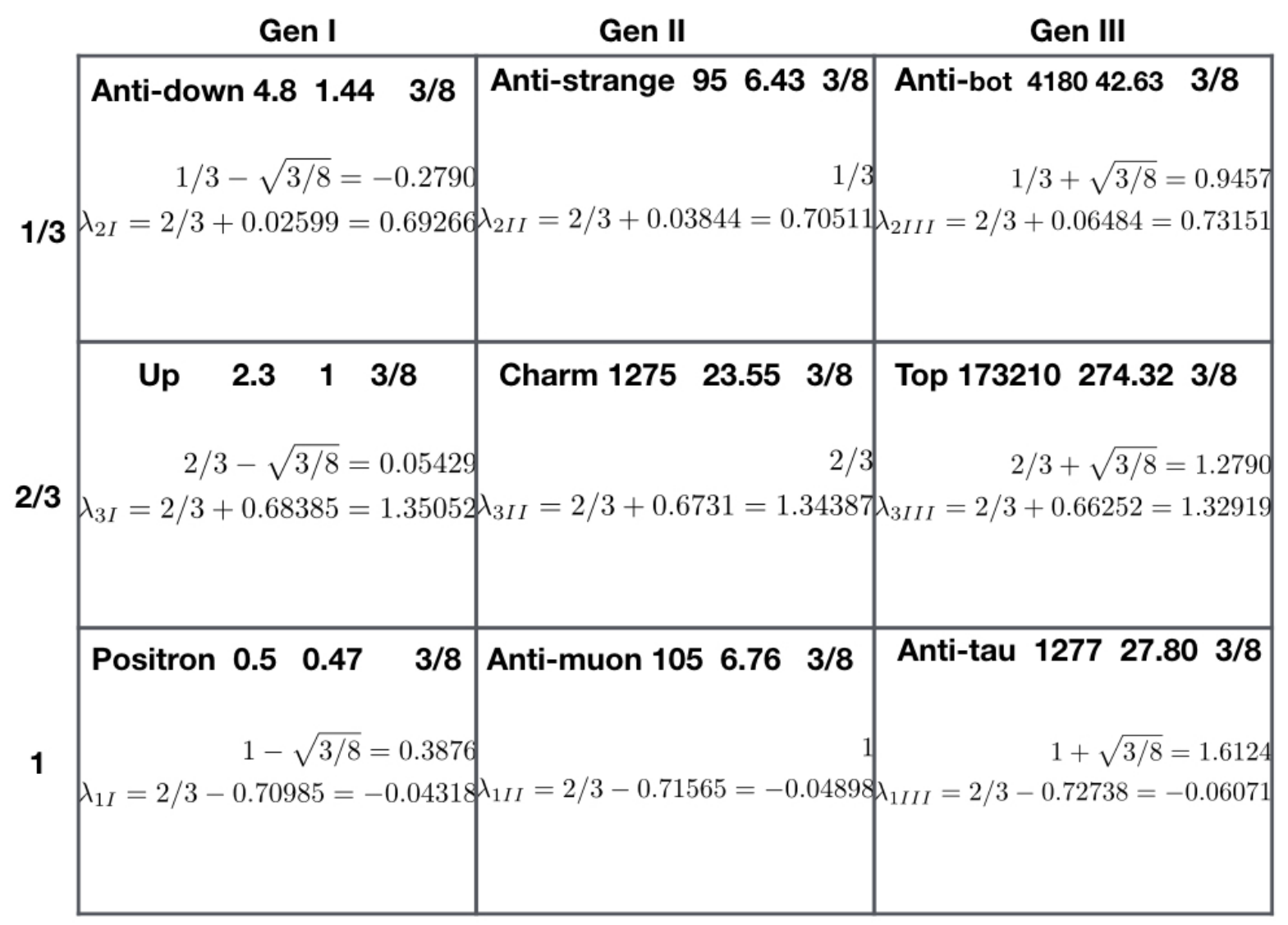

The Jordan Eigenvalues

FIG. 6. The eighteen Jordan eigenvalues for the six quarks and three charged leptons. In each cell, at the top is shown the name of the particle, its mass in $\mathrm{MeV}$, square-root of mass ratio with respect to up quark, and the octonionic magnitude. The three eigenvalues in any given row are calculated by making a triplet of like charges. These eigenvalues, dubbed as the horizontal roots, are shown as the first entry in each of the nine cells. The three eigenvalues in any given column are calculated by making a triplet of like generation charged fermions. These are the vertical roots, shown as the lower entry in each cell. There are two roots for every charged fermion. Only one out of the 18 roots is negative - this is the upper entry for the anti-down quark.

roots of the masses of the positron, up quark, and down quark possess the ratio $1: 2: 3$ and hence they can be assigned the 'square-root-mass numbers' $(1 / 3,2 / 3,1)$ respectively, these being in the inverse order as the ratios of their electric charge. The $e / \sqrt{m}$ ratios for the three particles then have the respective values $(3,1,1 / 3)$, whereas $e \sqrt{m}$ has the respective values $(1 / 3,4 / 9,1 / 3)$. The choice of square-root of mass as being more fundamental than mass is justified by recalling that in our approach, gravitation is derived from 'squaring' an underlying spin one Lorentz interaction [4]. It is reasonable then to assume that the spin one Lorentz interaction is sourced by $\sqrt{m}$, and to try to understand the origin of the square-root 
of the mass ratios, rather than origin of the mass ratios themselves.

At this stage, the above proposed quantised root-mass-ratios for the first generation are only an assumption; we do not have a proof for this assumption. [We return to thus aspect in detail in a forthcoming publication [42], where we consider an $S U(3)$ gravi-color symmetry for gravitation, analogous to $S U(3)_{\text {color }}$ for QCD, and actually demonstrate a square-root mass ratio 1:2:3 for electron, up quark and down quark.] A justification might come from the following. The automorphism group $G_{2}$ of the octonions has the two maximal subgroups $S U(3)$ and $S O(4)$. These two groups have an intersection $U(2) \sim S U(2) \times U(1)$. The $S U(3)$ is identified with $S U(3)_{c}$, the $S U(2)$ with the weak symmetry, and the $U(1)$ with $U(1)_{\text {em }}$. Thus the $U(1)_{e m}$ is a subset also of the maximal sub-group $S O(4)$ which led us to propose the Lorentz-Weak-Electro symmetry, and hence this $U(1)$ might also determine the said quantised root-mass-ratios $(1 / 3,2 / 3,1)$ for the positron, up quark, and down quark respectively. For now, we take these quantised root-mass-ratios as a working hypothesis. This implies, assuming a mass $0.511 \mathrm{MeV}$ for the electron, a consequent predicted mass of 2.044 $\mathrm{MeV}$ for the up quark, and a predicted mass 4.599 MeV for the down quark.

If we assume that the $e / \sqrt{m}$ ratios for the first generation of the charged fermions are absolute values [valid prior to the enormous scaling down of mass] then we can assign a root-mass number $e / 3$ to the positron [and hence a mass number $e^{2} / 9$ ], where the electric charge $e$ is as given in Eqn. (74). Hence the mass-number for the positron/electron is

$$
\sqrt{G_{N}} m_{e+}=(1 / 1024) \exp [2 / 9-1 / \sqrt{6}]\left(\hbar L_{P} / t_{P}\right)^{1 / 2}
$$

where $G_{N}$ is Newton's gravitational constant. Thus the mass number of the electron is $1 /(137 \times 9)$ of Planck mass and has to be scaled down by the factor $f=2 \times 10^{19}$ before it acquires the observed mass of $0.5 \mathrm{MeV}$. This then is also the universal factor by which the assigned mass number of every quark and charged lepton must be scaled down to get it to its current value. This is not far from the twenty orders of mass-scale-down by the Karolyhazy effect in cosmology, proposed earlier in this section. The initial ratio of the electrostatic to gravitational attraction between an electron and a positron is $e^{2} /\left(e^{4} / 81\right) \sim 137 \times 81 \sim 10^{4}$.

Now, to deduce the observed mass-ratios for the second and third generations, we recall 


\begin{tabular}{|c|c|c|}
\hline Gen I & Gen II & Gen III \\
\hline $\begin{array}{l}\text { Anti - down quark } \\
4.599 \quad 1 \\
5 \pi / 6 \\
\left(\theta_{I}+4 \pi\right) / 3\end{array}$ & $\begin{array}{l}\text { Anti - strange quark } \\
95 \pm 5 \quad 4.55 \\
9 \pi / 6 \\
\left(\theta_{I I}+4 \pi\right) / 3\end{array}$ & $\begin{array}{l}\text { Anti - bottom quark } \\
4180 \pm 30 \quad 30.15 \\
\pi / 6 \\
\left(\theta_{I I I}+4 \pi\right) / 3\end{array}$ \\
\hline $\begin{array}{l}\text { Up quark } \\
2.044 \quad 2 / 3 \\
5 \pi / 6 \\
\theta_{I} / 3\end{array}$ & $\begin{array}{l}\text { Charm quark } \\
1275 \pm 25 \quad 16.65 \\
9 \pi / 6 \\
\theta_{I I} / 3\end{array}$ & \begin{tabular}{|l} 
Top Quark \\
$173210 \pm 510 \pm 710 \quad 194.07$ \\
$\pi / 6$ \\
$\theta_{I I I} / 3$
\end{tabular} \\
\hline $\begin{array}{l}\text { Positron } \\
0.511 \quad 1 / 3 \\
5 \pi / 6 \\
\left(\theta_{I}+2 \pi\right) / 3\end{array}$ & $\begin{array}{l}\text { Anti }- \text { muon } \\
105.7 \quad 4.79 \\
9 \pi / 6 \\
\left(\theta_{I I}+2 \pi\right) / 3\end{array}$ & $\begin{array}{l}\text { Anti - tau Lepton } \\
1777 \quad 19.66 \\
\pi / 6 \\
\left(\theta_{I I I}+2 \pi\right) / 3\end{array}$ \\
\hline
\end{tabular}

\section{The Jordan Angles}

FIG. 7. The Jordan angles for the six quarks and the three charged leptons. In each cell the first row shows the mass of the particle in $\mathrm{MeV}$ and the square-root of the mass ratio taken with respect to the anti-down quark. The second row in each cell shows the Jordan angle from which the first set of eigenvalues are made [by clubbing like charges]. This eigenvalue is obtained by taking the cosine of the shown angle, multiplying it by $2 \sqrt{-Q}$, and adding the result to the electric charge value. The last row in each cell shows the angle using which the second set of eigenvalues [made by clubbing fermions of a given generation] are made. Here also the cosine of the angle is taken, multiplied by $2 \sqrt{-Q}$ and the result added to $2 / 3$. In terms of these two angles the nine fermions are placed symmetrically on a 2-torus; yet the angles manage to give rise to the measured mass ratios which appear to be quite random otherwise.

from above that the three generations are respectively characterised by these three angles

$$
\theta_{I}=1.81270 \sim 97^{\circ} ; \quad \theta_{I I}=1.69730 \sim 100^{\circ} ; \quad \theta_{I I I}=1.74837 \sim 104^{o}
$$

These three angles can be taken to be the defining characteristic of the three generations. All the three angles lie in the second quadrant and hence have a negative cosine; therefore the largest root $\lambda_{1}$ in (68) for each of the three generations is identified with the quark 
having $2 / 3$ charge [i.e. up, charm, top]. In Eqn. (73) these are roots $\left(\lambda_{3 I}, \lambda_{3 I I}, \lambda_{3 I I I}\right)$ for the up, charm and top respectively. In Gen I, the next root is derived by taking the angle $\left(\theta_{I}+2 \pi\right) / 3=2.45 \sim 140^{\circ}$ which lies in the second quadrant, and gives the smallest root $\lambda_{1 I}$ which is assigned to the positron. The third root $\lambda_{2 I}$ comes from taking the angle $(\theta+4 \pi) / 3=4.54 \sim 260^{\circ}$ which lies in the third quadrant. So one moves from the up quark to the positron to the anti-down quark while going from the first to the second to the third quadrant. In Gen II, the second root $\lambda_{1 I I}$ comes from the angle $\left(\theta_{I I}+2 \pi\right) / 3=$ $2.47 \sim 141^{0}$ and is assigned to the anti-muon, whereas the third root $\lambda_{2 I I}$ coming from the angle $\left(\theta_{I I}+4 \pi\right) / 3=4.56 \sim 261^{\circ}$ is assigned to the anti-strange quark. In GenIII the second root $\lambda_{1 I I I}$ coming from the angle $\left(\theta_{I I I}+2 \pi\right) / 3=2.57 \sim 147^{\circ}$ is assigned to the tau-lepton, whereas the third root coming from the angle $\left(\theta_{I I I}+4 \pi\right) / 3=4.66 \sim 267^{\circ}$ is for the anti-bottom quark.

We can place the six quarks and three charged leptons on a two-torus, and identify each one of them with a pair of angles on the torus (one angle along each of the two independent directions). We have already identified these angles corresponding to the second set of eigenvalues, in the previous paragraph. Similarly, we can evaluate the angles corresponding to the first set of eigenvalues, found in Section II, and listed in the table in Fig. 5, by comparing those roots with their equivalent angular form given in Eqn. (68). For the three neutrinos, we conclude from the roots given in $(29)$, that the three angles are $(\pi / 6,5 \pi / 6,9 \pi / 6)$. The same angles also arise for the charged fermions, with the first angle for the GenIII particle, next one for GenI and largest angle for GenII. Also, in each case, $R=0$, while $-Q=1 / 8$. The table in Figure 6 below shows these Jordan angles, along with the measured mass values, as well the square-root of the mass ratio taken with respect to mass of the anti-down quark. We now see that the nine fermions are placed symmetrically on the torus, as far as the angles are concerned. And yet these angles manage to give rise to strange-looking mass ratios.

Since the square-root-mass ratio of the anti-down quark has been set to unity, and predicted above to be $4.599 \mathrm{MeV}(=9 \times 0.511 \mathrm{MeV})$, we will calculate the square-root-mass ratios of the other particles with respect to the anti-down-quark, and demonstrate a correlation of these ratios with the Jordan eigenvalues. Also, since a negative Jordan eigenvalue is to be associated with minus of square-root mass, for finding the mass-ratio, we take the absolute value of the anti-down-quark eigenvalue, which is negative. 
- Anti-muon : Take the ratio of the first set of Jordan eigenvalues for the electron and the muon [see the table in Fig. 6]. Multiply by a factor representing the down quark (the first factor in the expression below). Then compare the resulting value with the square-root mass ratio of the muon mass with respect to the electron mass:

$$
\frac{1+\sqrt{3 / 8}}{1-\sqrt{3 / 8}} \times \frac{1 / 3+\sqrt{3 / 8}}{|1 / 3-\sqrt{3 / 8}|}=14.10 ; \quad \sqrt{206.7682830}=14.38
$$

- Anti-tau lepton : Using the first set of eigenvalues for the charged leptons, we get the ratio for tau-lepton to electron:

$$
\frac{1+\sqrt{3 / 8}}{1-\sqrt{3 / 8}} \times \frac{1 / 3+\sqrt{3 / 8}}{|1 / 3-\sqrt{3 / 8}|} \times \frac{1+\sqrt{3 / 8}}{1-\sqrt{3 / 8}}=58.64 ; \sqrt{\frac{1776.86}{.511}}=58.97
$$

- Charm quark with respect to up quark: This ratio is same as the ratio of charm / up in Eqn. (38).

$$
\frac{2 / 3+\sqrt{3 / 8}}{2 / 3-\sqrt{3 / 8}}=23.57 ; \quad \sqrt{\frac{1275}{2.3}}=23.55
$$

- Top quark with respect to up quark: Again this ratio is analogous to the one for top / up in Eqn. (40).

$$
\frac{2 / 3+\sqrt{3 / 8}}{2 / 3-\sqrt{3 / 8}} \times \frac{2 / 3}{2 / 3-\sqrt{3 / 8}}=289.26 ; \quad \sqrt{\frac{173210}{2.3}}=274.42
$$

- Anti-strange quark with respect to down quark:

$$
\frac{1+\sqrt{3 / 8}}{1-\sqrt{3 / 8}} \times 1=4.20 ; \quad \sqrt{\frac{95}{4.7}}=4.50
$$

- Anti-bottom quark with respect to down quark:

$$
\frac{1+\sqrt{3 / 8}}{1-\sqrt{3 / 8}} \times \frac{1+\sqrt{3 / 8}}{1} \times \frac{1+\sqrt{3 / 8}}{1-\sqrt{3 / 8}}=28.44 ; \quad \sqrt{\frac{4180}{4.7}}=29.82
$$

These ratios made from the Jordan eigenvalues suggest a possible correlation with the squareroot mass ratios, and hence provide a plausible definition of a mass quantum number for standard model fermions. This definition is completely independent of trace dynamics and 
its Lagrangian, and is a property exclusively of the octonionic algebra. This is completely analogous to the fact that in the octonionic approach to the standard model, quantisation of electric charge is deduced from eigenvalues of the $U(1)_{e} m$ operator made from the Clifford algebra $C l(6)$. Hence, square-root of mass is treated on the same footing as electric charge: their quantisation is a property of the algebra, not of the dynamics. The difference between charge quantisation and mass quantisation is that for finding the mass eigenstates, all three generations must be considered together, not one at a time.

The square-root mass numbers for the charged fermions are shown in Fig. 8. These have the same fundamental status as quantised electric charge values $1 / 3,2 / 3$ and 1 .

\section{Square-root mass numbers for charged fermions}

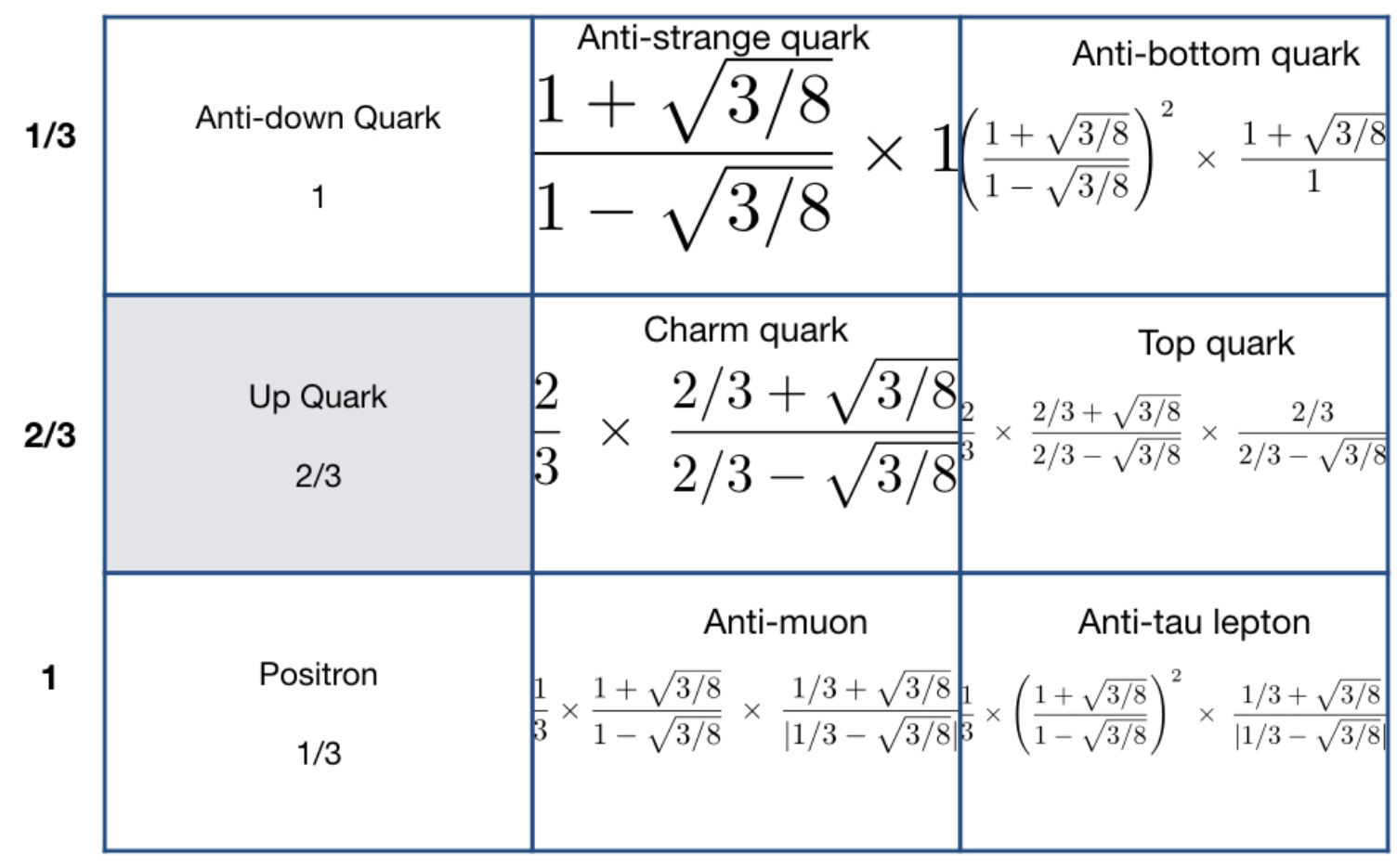

Electric charge for a given row shown on its left

FIG. 8. The square-root mass numbers for charged fermions. These have the same fundamental status as quantised electric charge values $1 / 3,2 / 3$ and 1 . 


\section{Quantum non-locality}

Additional internal spatial dimensions which are not compact, yet very thin, offer a promising resolution to the quantum non-locality puzzle, thereby lifting the tension with 4D special relativity. Let us consider once again Baez's cube of Fig. 3. Any of the three quaternionic spaces containing the unit element 1 can play the role of the emergent 4D classical space-time in which classical systems evolve. Let us say this classical universe is the plane $\left(1 e_{6} e_{1} e_{5}\right)$. Now, the true universe is the full $8 \mathrm{D}$ octonionic universe, with the four internal dimensions being probed [only by] quantum systems. Now we must recall that these four internal dimensions are extremely thin, of the order of Fermi dimensions, and along these directions no point is too far from each other, even if their separation in the classical 4D quaternion plane is billions of light years! Consider then, that Alice at 1 and Bob at $e_{1}$ are doing space-like separated measurements on a quantum correlated pair. Whereas the event at $e_{1}$ is outside the light cone of 1 , the correlated pair is always within each other's quantum wavelength along the internal directions, say the path $\left(1 e_{3} e_{2} e_{7} e_{1}\right)$. The pair influences each other along this path acausally, because this route is outside the domain of 4D Lorentzian spacetime and its causal light-cone structure. The internal route is classically forbidden but allowed in quantum mechanics. This way neither special relativity nor quantum mechanics needs to be modified. It is also interesting to ask if evolution in Connes time in this 8D octonionic universe obeying generalised trace dynamics can violate the Tsirelson bound.

The exceptional Jordan algebra is of significance also in superstring theory, where it has been suggested that there is a relation between the EJA and the vertex operators of superstrings, and that the vertex operators represent couplings of strings [43, 44]. This intriguing connection between the EJA, string theory and aikyon theory deserves to be explored further.

Lastly we mention that the Lagrangian (45) that we have been studying closely resembles the Bateman oscillator [45] model, for which the Lagrangian is

$$
L=m \dot{x} \dot{y}+\gamma(x \dot{y}-\dot{x} y)-k x y
$$

I thank Partha Nandi for bringing this fact to my attention. Considering that the Bateman oscillator represents a double oscillator with relative opposite signs of energy for the two 
oscillators undergoing damping, it is important to understand the implications for our theory. In particular, could this imply a cancellation of zero point energies between bosonic and fermionic modes, thus annulling the cosmological constant? And also whether this damping is playing any possible role in generating matter-anti-matter asymmetry?

Acknowledgements: I would like to thank Carlos Perelman for discussions and helpful correspondence, and for making me aware of the beautiful work of Dray and Manogue on the Jordan eigenvalue problem. I also thank Vivan Bhatt, Tanmoy Bhattacharya, Cohl Furey, Niels Gresnigt, Garrett Lisi, Nehal Mittal, Rajrupa Mondal, Roberto Onoforio, Robert Wilson and Vatsalya Vaibhav for useful correspondence and discussions.

\section{APPENDIX: PHYSICAL MOTIVATION FOR THE PRESENT THEORY: QUANTUM (FIELD) THEORY WITHOUT CLASSICAL TIME, AS A ROUTE TO QUANTUM GRAVITY AND UNIFICATION}

In this appendix, we recall from earlier work [4] the motivation for developing a formulation of quantum theory without classical time, and how doing so leads to a pre-quantum, prespacetime theory which is a candidate for unification of general relativity with the standard model.

A. Why there must exist a formulation of quantum theory which does not refer to classical time? And why such a formulation must exist at all energy scales, not just at the Planck energy scale.

Classical time, on which quantum systems depend for a description of their evolution, is part of a classical space-time. Such a space-time - the manifold as well as the metric that overlies it - is produced by macroscopic bodies. These macroscopic bodies are a limiting case of quantum systems. In principle one can imagine a universe in which there are no macroscopic bodies, but only microscopic quantum systems. And this need not be just at the Planck energy scale.

As a thought experiment, consider an electron in a double slit interference experiment, having crossed the slits, and not yet reached the screen. It is in a superposed state, as 
if it has passed through both the slits. We want to know, non-perturbatively, what is the spacetime geometry produced by the electron? Furthermore, we imagine that every macroscopic object in the universe is suddenly separated into its quantum, microscopic, elementary particle units. We have hence lost classical space-time! Perturbative quantum gravity is no longer possible. And yet we must be able to describe what gravitational effect the electron in the superposed state is producing. This is the sought for quantum theory without classical time! And the quantum system is at low non-Planckian energies, and is even non-relativistic. This is the sought for formulation we have developed, assuming only three fundamental constants a priori: Planck length $L_{P}$, Planck time $t_{P}$, and Planck's constant $\hbar$. Every other dimensionful constant, e.g. electric charge, and particle masses, are expressed in terms of these three. This new theory is a pre-quantum, pre-spacetime theory, needed even at low energies.

A system will be said to be a Planck scale system if any dimensionful quantity describing the system and made from these three constants, is order unity. Thus if time scales of interest to the system are order $t_{P}=10^{-43} \mathrm{~s}$, the system is Planckian. If length scales of interest are order $L_{P}=10^{-33} \mathrm{~cm}$, the system is Planckian. If speeds of interest are of the order $L_{P} / t_{P}=c=3 \times 10^{8} \mathrm{~cm} / \mathrm{s}$ then the system is Planckian. If the energy of the system is of the order $\hbar / t_{P}=10^{19} \mathrm{GeV}$, the system is Planckian. If the action of the system is of the order $\hbar$, the system is Planckian. If the charge-squared is of the order $\hbar c$, the system is Planckian. Thus in our concepts, the value 1/137 for the fine structure constant, being order unity in the units $\hbar c$, is Planckian. This explains why this pre-quantum, pre-spacetime theory knows the low energy fine structure constant.

A quantum system on a classical space-time background is hugely non-Planckian. Because the classical space-time is being produced by macroscopic bodies each of which has an action much larger than $\hbar$. The quantum system treated in isolation is Planckian, but that is strictly speaking a very approximate description. The spacetime background cannot be ignored only when the background is removed from the description, the system is exactly Planckian. This is the pre-quantum, pre-spacetime theory.

It is generally assumed that the development of quantum mechanics, started by Planck in 1900, was completed in the 1920s, followed by generalisation to relativistic quantum field theory. This assumption, that the development of quantum mechanics is complete, is not necessarily correct - quantisation is not complete until the last of the classical elements - 
this being classical space-time - has been removed from its formulation.

The pre-quantum, pre-spacetime theory achieves that, giving also an anticipated theory of quantum gravity. What was not anticipated was that removing classical space-time from quantum theory will also lead to unification of gravity with the standard model. And yield an understanding of where the standard model parameters come from. It is clear that the sought for theory is not just a high energy Beyond Standard Model theory. It is needed even at currently accessible energies, so at to give a truly quantum formulation of quantum field theory. Namely, remove classical time from quantum theory, irrespective of the energy scale. Surprisingly, in doing so, we gain answers to unsolved low energy aspects of the standard model and of gravitation.

The process of quantisation works very successfully for non-gravitational interactions, because they are not concerned with space-time geometry. However, it is not necessarily correct to apply this quantisation process to spacetime geometry. Because the rules of quantum theory have been written by assuming a priori that classical time exists. How then can we apply these quantisation rules to classical time itself? Doing so leads to the notorious problem of time in quantum gravity - time is lost, understandably. We do not quantise gravity. We remove classical space-time / gravity from quantum [field] theory. Space-time and gravity emerge as approximations from the pre-theory, concurrent with the emergence of classical macroscopic bodies. In this emergent universe, those systems which have not become macroscopic, are described by the beloved quantum theory we know namely quantum theory on a classical spacetime background. This is an approximation to the pre-theory: in this approximation, the contribution of the said quantum system to the background spacetime is [justifiably] neglected.

B. Why a quantum theory of gravity is needed at all energy scales, and not just at the Planck energy scale? And how that leads us to partially redefine what is meant by Planck scale: Replace Energy by Action.

We have argued above that there must exist a formulation of quantum theory which does not refer to classical time. Such a formulation must in principle exist at all energy scales, not just at the Planck energy scale. For instance, in today's universe, if all classical objects were to be separated out into elementary particles, there would be no classical space-time 
and we would need such a formulation. Even though the universe today is a low energy universe, not a Planck energy universe.

Such a formulation is inevitably also a quantum theory of gravity. Arrived at, not by quantising gravity, but by removing classical gravity from quantum theory. We can also call such a formulation pure quantum theory, in which there are no classical elements: classical space-time has been removed from quantum theory. We also call it a pre-quantum, prespacetime theory.

What is meant by Planck scale, in this pre-theory?

Conventionally, a phenomenon is called Planck scale if: the time scale $T$ of interest is of the order Planck time $t_{P}$; and/or length scale $L$ of interest is of the order of Planck length $L_{P}$; and/or energy scale $E$ of interest is of the order Planck energy $E_{P}$. According to this definition of Planck scale, a Planck scale phenomenon is quantum gravitational in nature. Since the pre-theory is quantum gravitational, but not necessarily at the Planck energy scale, we must partially revise the above criterion, when going to the pre-theory: replace the criterion on energy $E$ by a criterion on something else. This something else being the action of the system!

In the pre-theory, a phenomenon is called Planck scale if: the time scale $T$ of interest is of the order Planck time $T_{P}$; and/or length scale $L$ of interest is of the order of Planck length $L_{P}$; and/or the action $S$ of interest is of the order Planck constant $\hbar$. According to this definition of Planck scale, a Planck scale phenomenon is quantum gravitational in nature.

Why does this latter criterion make sense? If every degree of freedom has an associated action of order $\hbar$, together the many degrees of freedom cannot give rise to a classical spacetime. Hence, even if the time scale $\mathrm{T}$ of interest and length scale $\mathrm{L}$ of interest are NOT Planck scale, the system is quantum gravitational in nature. The associated energy scale $\hbar / T$ for each degree of freedom is much smaller than Planck scale energy $E_{P}$. Hence in the pre-theory the criterion for a system to be quantum gravitational is DIFFERENT from conventional approaches to quantum gravity. And this makes all the difference to the formulation and interpretation of the theory. e.g. the low energy fine structure constant $1 / 137$ is a Planck scale phenomenon [according to the new definition] because the square of the electric charge is order unity in the units $\hbar c=\hbar L_{P} / t_{P}$

In our pre-theory, there are three, and only three, fundamental constants: Planck length $L_{P}$, Planck time $t_{P}$ and Planck action $\hbar$. Every other parameter, such as electric charge, 
Newton's gravitational constant, standard model coupling constants, and masses of elementary particles, are defined and derived in terms of these three constants: $\hbar, L_{P}$ and $t_{P}$.

In the pre-theory the universe is an $8 \mathrm{D}$ octonionic universe, as shown in the Fig. 3, the octonion, reproduced below. The origin $e_{0}=1$ stands in for the real part of the octonion [coordinate time] and the other seven vertices stand in for the seven imaginary directions. A degree of freedom [i.e. 'particle' or an atom of space-time-matter (STM)] is described by a matrix $q$ which resides on the octonionic space: $q$ has eight coordinate components $q_{i}$ where each $q_{i}$ is a matrix. We have replaced a four-vector in Minkowski space-time by an eightmatrix in octonionic space: and this describes the particle / STM atom. The STM atom evolves in Connes time, this time being over and above the eight octonionic coordinates. Its action is that of a free particle in this space: time integral of kinetic energy, the latter being the square of velocity $\dot{q}$, where dot is derivative with respect to Connes time. Eight octonionic coordinates are equivalent to ten Minkowski coordinates, because of $S L(2, O) \sim \operatorname{Spin}(9,1)$. The symmetries of this space are the symmetries of the (complexified) octonionic algebra: they contain within them the symmetries of the standard model, including the 4D-Lorentz symmetry.

The classical 4D Minkowski universe is one of the three planes (quaternions) intersecting at the origin $e_{0}=1$. Incidentally the three lines originating from $e_{0}$ represent complex numbers. The four imaginary directions not connected to the origin represent directions along which the standard model forces lie (internal symmetries). Classical systems live on the 4D quaternionic plane. Quantum systems (irrespective of whether they are at Planck energy scale) live on the entire $8 \mathrm{D}$ octonion. Their dynamics is the sought for quantum theory without classical time. This dynamics is oblivious to what is happening on the $4 \mathrm{D}$ classical plane. QFT as we know it is this pre-theory projected to the 4D Minkowski space-time. The present universe has arisen as a result of a symmetry breaking in the 8D octonionic universe: the electroweak symmetry breaking. Which in this theory is actually the color-electro - weak-Lorentz symmetry breaking. Classical systems condense on to the 4D Minkowski plane as a result of spontaneous localisation, which precipitates the electroweak symmetry breaking in the first place. The fact that weak is part of weak-lorentz should help understand why the weak interaction violates parity, whereas electro-color does not. Hopefully the theory will shed some light also on the strong-CP problem. 


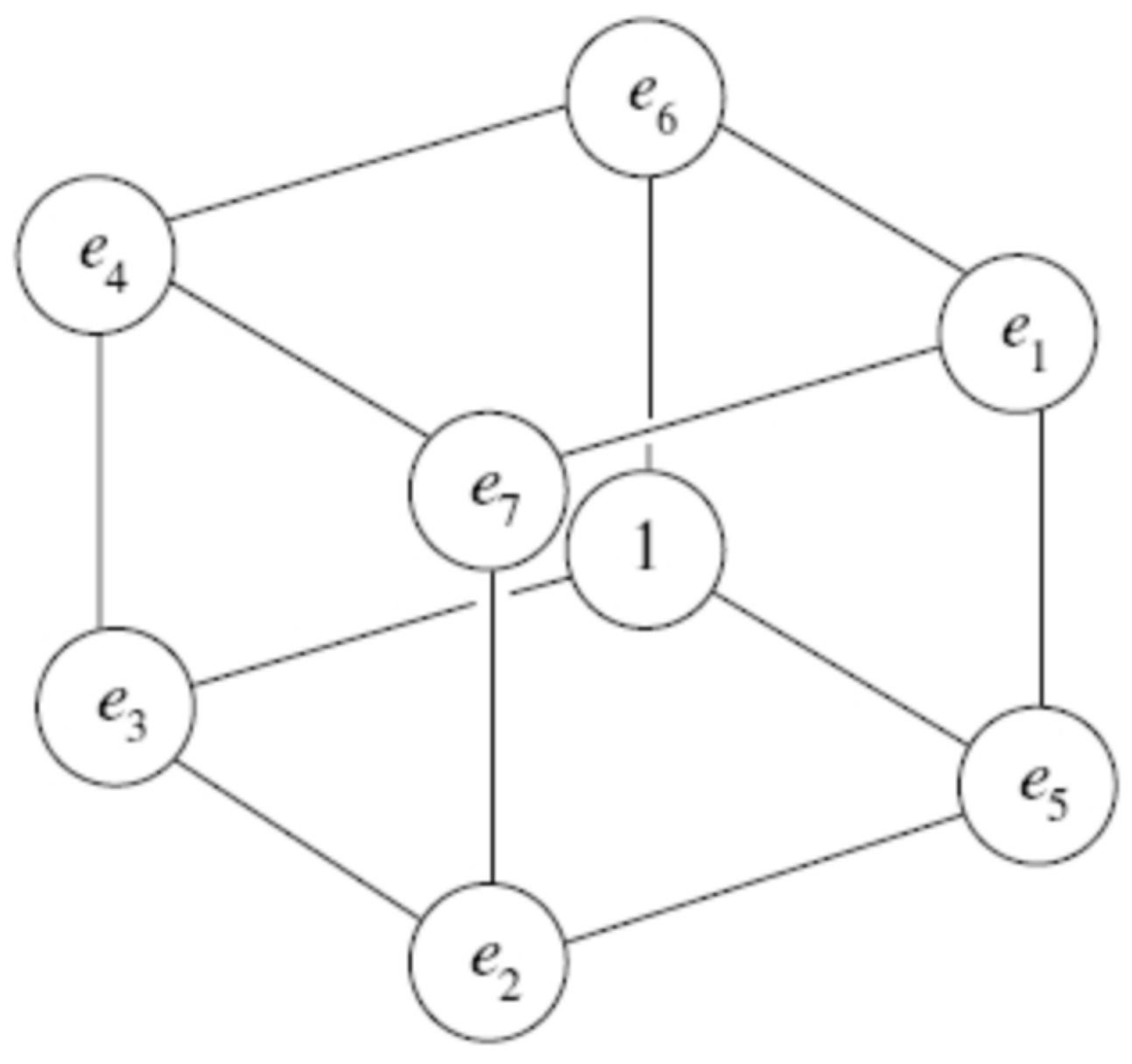

FIG. 9. The octonions [From Baez [23]].

C. What is Trace Dynamics? : Trace dynamics is quantisation, without imposing the Heisenberg algebra

In the conventional development of canonical quantisation, the two essential steps are:

1. Quantisation Step 1 is to raise classical degrees of freedom, the real numbers $q$ and $p$, to the status of operators / matrices. This is a very reasonable thing to do.

2. Quantisation Step 2 is very restrictive! Impose the Heisenberg algebra $[q, p]=i \hbar$. Its only justification is that the theory it gives rise to is extremely successful and consistent with every experiment done to date. In classical dynamics, the initial values of $q$ and $p$ are independently prescribed. There is NO relation between the initial $q$ and $p$. Once prescribed initially, their evolution is determined by the dynamics. Whereas, in quantum mechanics, a 
theory supposedly more general than classical mechanics, the initial values of the operators $q$ and $p$ must also obey the constraint $[q, p]=i \hbar$. This is highly restrictive!

3. It would be more reasonable if there were to be a dynamics based only on Quantisation Step 1. And then Step 2 emerges from this underlying dynamics in some approximation. This is precisely what Trace Dynamics is. Only step 1 is applied to classical mechanics. $q$ and $p$ are matrices, and the Lagrangian is the trace of a matrix polynomial made from $q$ and its velocity. The matrix valued equations of motion follow from variation of the trace Lagrangian. They describe dynamics. This is the theory of trace dynamics developed by Adler [1-3] - a pre-quantum theory, which we have generalised to a pre-quantum, prespacetime theory [10].

4. This matrix valued dynamics, i.e. trace dynamics, is more general than quantum field theory, and assumed to hold at the Planck scale, and also whenever background classical spacetime is absent, no matter what the energy scale. The Heisenberg algebra is shown to emerge at lower energies, or when space-time emerges, after coarse-graining the trace dynamics over length scales much larger than Planck length scale. Thus, quantum theory is midway between trace dynamics and classical dynamics.

5. The moral of the story is that we assume that quantum field theory does not hold at the Planck scale. Trace dynamics does. QFT is emergent.

6. The other assumption one makes at the Planck scale is to replace the 4-D classical spacetime manifold by an 8D octonionic spacetime manifold, so as to obtain a canonical definition of spin. This in turn allows for a Kaluza-Klein type unification of gravity and the standard model. Also, an 8D octonionic spacetime is equivalent to a 10-D Minkowski space-time. It is very rewarding to work with 8D octonionic, rather than 10D Minkowski the symmetries manifest much more easily.

7. Trace dynamics plus octonionic spacetime together give rise to a highly promising avenue for constructing a theory of quantum gravity, and of unification. 4D classical spacetime obeying GR emerges as an approximation at lower energies, alongside the emergent quantum theory.

8. How is this different from string theory? In many ways it IS like string theory, but without the Heisenberg algebra! The gains coming from dropping $[q, p]=i \hbar$ at the Planck scale are enormous. One now has a non-perturbative description of pre-space-time at the Planck scale. The symmetry principle behind the unification is very beautiful: physical 
laws are invariant under algebra automorphisms of the octonions. This unifies the internal gauge transformations of the standard model with the 4D spacetime diffeomorphisms of general relativity. The automorphism group of the octonions, the Lie group $G_{2}$, which is the smallest of the five exceptional Lie groups, contains within itself the symmetries $S U(3) \times S U(2) \times U(1)$ of the standard model, along with the Lorentz symmetry. The free parameters of the standard model are determined by the characteristic equation of the exceptional Jordan algebra $J_{3}(O)$, whose automorphism group $F_{4}$ is the exceptional Lie group after $G_{2}$.

\section{Normed division algebras, trace dynamics, and relativity in higher dimensions.}

\section{And how these relate to quantum field theory and the standard model}

Let us consider the four normed division algebras $\mathbb{R}, \mathbb{C}, \mathbb{H}, \mathbb{O}$ [Reals, Complex Numbers, Quaternions, Octonions] in the context of the space-times associated with them, and how these algebras relate to trace dynamics. This can be understood graphically with the help of Fig. 7 above, which contains within itself a representation of all the four division algebras.

Let us start with the reals $\mathbb{R}$, represented in the above diagram by the origin $e_{0}=1$. This direction represents the time coordinate, in all the four different space-times associated with these four division algebras. The three lines emanating from the origin and connecting respectively to $e_{3}, e_{5}, e_{6}$ represent complex numbers $\mathbb{C}$. The three planes intersecting at the origin represent quaternions $\mathbb{H}$ and the full cube represents the octonions $\mathbb{O}$.

Galilean relativity and Newtonian mechanics: This is related to the quaternions, and we assume each of the three planes intersecting at the origin represent absolute Newtonian space [say in the plane $\left(1, e_{1}, e_{5}, e_{6}\right)$ we set $\left.e_{1}=\hat{x}, e_{5}=\hat{y}, e_{6}=\hat{z}\right]$. Galilean invariance is assumed, and the spatial symmetry group is $S O(3)$, the group of rotations in three dimensional space; this is also the automorphism group $A u t(\mathbb{H})$ of the quaternions. The origin represents absolute Newtonian time, and we have Newtonian dynamics in which the action principle for the free particle represented by the configuration variable $\mathbf{q}$, which is a three-vector, is simply

$$
S=\int d t \dot{\mathbf{q}}^{2}
$$

The generalisation to many-particle systems interacting via potentials is obvious and well- 
known. Newtonian gravity can be consistently described in this framework. The dynamical variables, being real-number valued three-vectors, all commute with each other. The important approximation made in the physical space is that by hand we set $e_{1}^{2}=e_{2}^{2}=e_{3}^{2}=1$, instead of -1 . This of course is what gives us the Newtonian absolute space (Euclidean geometry) and absolute time, and the manifold $R^{3}$ for physical space. The associated algebra is $\mathbb{R} \times \mathbb{H}$, in an approximate sense, which becomes precise only in special relativity, as discussed below.

[The algebra $\mathbb{C}$ represents a $2 \mathrm{D}$ physical space, and $\mathbb{R} \times \mathbb{C}$ represents a space-time for Newtonian mechanics in absolute two-space represented by $\mathbb{C}$, and absolute time $\mathbb{R}$. The homomorphism $S L(2, \mathbb{R}) \sim S O(2,1)$ suggests that we can relate $2 \times 2$ real-valued matrices to a $2+1$ relativistic space-time. This observation becomes very relevant when we relate normed division algebras to relativity.]

To go from here to trace dynamics, we will raise all dynamical variables from three-vectors to three-matrices. Thus $\hat{\mathbf{q}}$ is a matrix-valued three-vector whose three spatial components $\hat{\mathbf{q}}_{1}, \hat{\mathbf{q}}_{2}, \hat{\mathbf{q}}_{3}$ are matrices whose entries are real numbers. The Lagrangian for a free particle will now be the trace of the matrix polynomial $\dot{\hat{\mathbf{q}}}^{2}$, and hence the action is

$$
S=\int d t \operatorname{Tr}\left[\dot{\hat{\mathbf{q}}}^{2}\right]
$$

The underlying three-space continues to have the symmetry group $S O(3)$ and the dynamics obeys Galilean invariance; this is implemented on the trace dynamics action via the unitary transformations generated by the generators of $S O(3)$.

Special relativity, Complex quaternions, and the algebra $\mathbb{R} \times \mathbb{C} \times \mathbb{H}$ :

Consider the quaternionic four vector $\mathbf{x}=x_{0} e_{0}+x_{1} e_{1}+x_{2} e_{2}+x_{4} e_{4}$ and the corresponding position four-vector for a particle in special relativity: $\mathbf{q}_{\mathbf{i}}=q_{0} e_{0}+q_{1} e_{1}+q_{2} e_{2}+q_{4} e_{4}$. One can define the four-metric on this Minkowski space-time whose symmetry group is the Lorentz group $S O(3,1)$ having the universal cover $\operatorname{Spin}(3,1)$ isomorphic to $S L(2, C)$. The complex quaternions generate the boosts and rotations of the Lorentz group $\mathrm{SO}(3,1)$. They can be used to obtain a faithful representation of the Clifford algebra $\mathrm{Cl}(2)$ and fermionic ladder operators constructed from this algebra can be used to generate the Lorentz algebra $S L(2, \mathbb{C})$. Also, $C l(2)$ can be used to construct left and right handed Weyl spinors as minimal left ideals of this Clifford algebra, and as is well known the Dirac spinor and the 
Majorana spinor can be defined from the Weyl spinors. $\mathrm{Cl}(2)$ also gives the vector and scalar representations of the Lorentz algebra. These results are lucidly described in Furey's Ph. D. thesis [6-8] as well as also in her video lecture series on standard model and division algebras https://www.youtube.com/watch?v=GJCKCss43WI\&ab channel=CohlFureyCohlFurey

The above relation between the Clifford algebra $C l(2)$ and the Lorentz algebra $S L(2, C)$ strongly suggests, keeping in view the earlier conclusions for $C l(6)$ and the standard model and the octonions [6-8], that the $C l(2)$ algebra describes the left handed neutrino and the right-handed anti-neutrino, and a pair of spin one Lorentz bosons. This is confirmed by writing the following trace dynamics Lagrangian and action on the quaternionic space-time of special relativity, thereby generalising the relativistic particle $S=-m c \int d s$ :

$$
\frac{S}{C_{0}}=\frac{a_{0}}{2} \int \frac{d \tau}{\tau_{P l}} \operatorname{Tr}\left[\dot{q}_{B}^{\dagger}+i \frac{\alpha}{L} q_{B}^{\dagger}+a_{0} \beta_{1}\left(\dot{q}_{F}^{\dagger}+i \frac{\alpha}{L} q_{F}^{\dagger}\right)\right] \times\left[\dot{q}_{B}+i \frac{\alpha}{L} q_{B}+a_{0} \beta_{2}\left(\dot{q}_{F}+i \frac{\alpha}{L} q_{F}\right)\right]
$$

where $a_{0} \equiv L_{P}^{2} / L^{2}$. This Lagrangian is identical in form to the one studied earlier in the present paper, but with a crucial difference that it is now written on $4 \mathrm{D}$ quaternionic spacetime, not on $8 \mathrm{D}$ octonionic space-time. Thus $\dot{q}_{B}$ and $q_{B}$ have four components between them, not eight: $q_{B}=q_{B e 2} e_{2}+q_{B e 4} e_{4} ; \dot{q}_{B}=\dot{q}_{B e 0} e_{0}+\dot{q}_{B e 1} e_{1}$. Similarly, the fermionic matrices have four components between them, not eight. Thus $q_{F}=q_{F e 2} e_{2}+q_{F e 4} e_{4} ; \dot{q}_{F}=$ $\dot{q}_{F e 0} e_{0}+\dot{q}_{F e 1} e_{1}$

This has far-reaching consequences. Consider first the case where we set $\alpha=0$. The Lagrangian then is

$$
\frac{S}{C_{0}}=\frac{a_{0}}{2} \int \frac{d \tau}{\tau_{P l}} \operatorname{Tr}\left[\dot{q}_{B}^{\dagger}+a_{0} \beta_{1} \dot{q}_{F}^{\dagger}\right] \times\left[\dot{q}_{B}+a_{0} \beta_{2} \dot{q}_{F}\right]
$$

By opening up the terms into their coordinate components, the various degrees of freedom can be identified with the Higgs, the Lorentz bosons, the neutral weak isospin boson, and two neutrinos. The associated space-time symmetry is the Lorentz group $S O(3,1)$ and the associated Clifford algebra is $C l(2)$, reminding us again of the homomorphism $S L(2, \mathbb{C}) \sim$ $S O(3,1)$.

When $\alpha$ is retained, the Lagrangian describes Lorentz-weak symmetry of the leptons: electron, positron, two neutrinos of the first generation, the Higgs, two Lorentz bosons, and the three weak isospin bosons. To our understanding, the associated Clifford algebra is still 
$C l(2)$ but now all the quaternionic degrees of freedom have been used in the Lagrangian and in the construction of the particle states.. What we likely have here is the extension of the Lorentz algebra by an $S U(2)$, as shown in Figure 8 below, borrowed from our earlier work [4]. It remains to be understood if now the homomorphism $S L(2, \mathbb{H}) \sim S O(5,1)$ comes into play. And also, whether a quaternionic triality [46] could explain the existence of three generations of leptons. These aspects are currently under investigation.

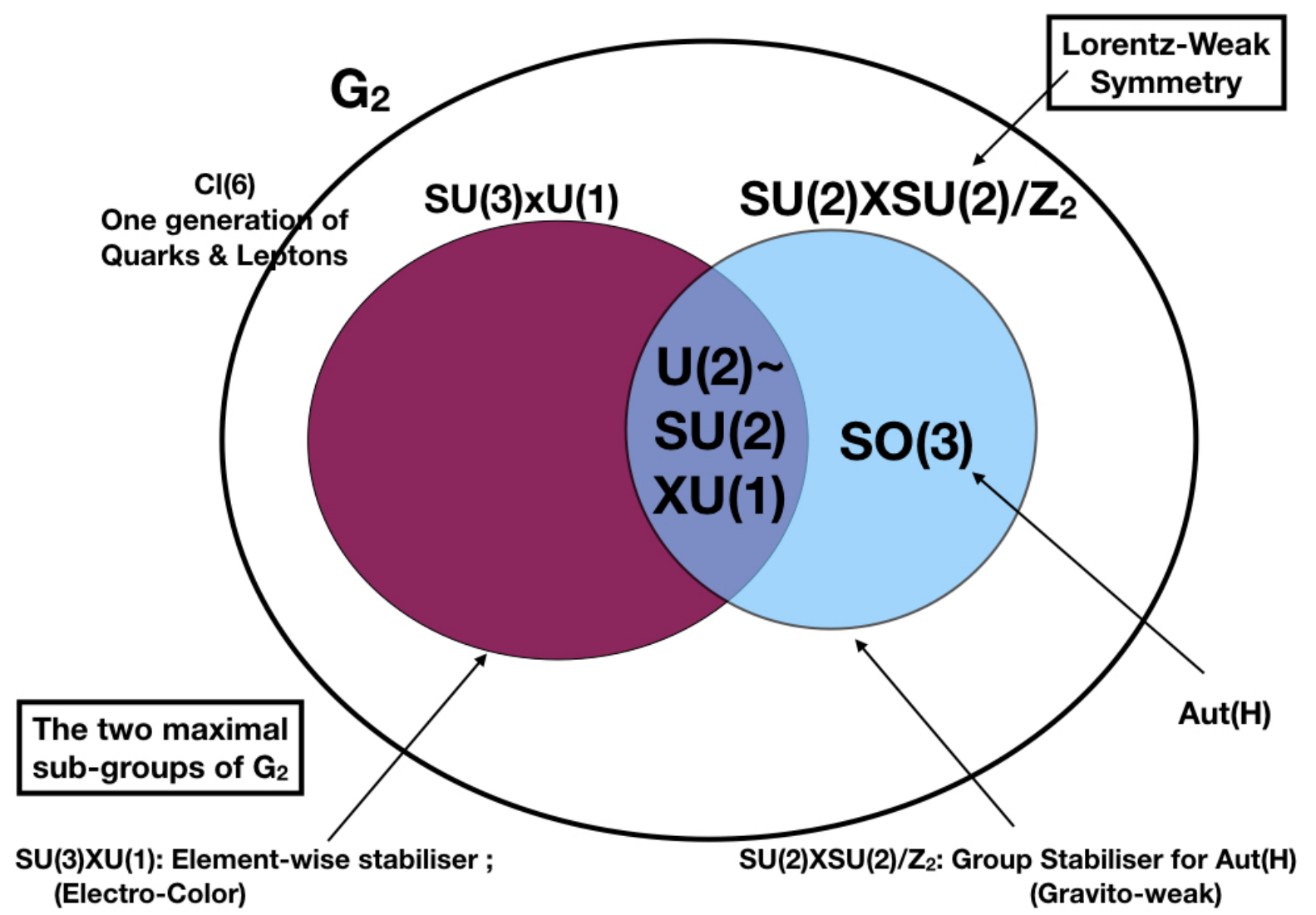

$\mathrm{U}(2)$ : Intersection of Electro-color and Gravito-Weak, SO(3): Aut(H) : To-be-Lorentz symmetry

FIG. 10. The maximal sub-groups of $G_{2}$ and their intersection [From Singh [4]].

It is now only natural that this trace dynamics be extended to the last of the division algebras, the octonions, so as to construct an octonionic special relativity. This amounts to extending the Lorentz algebra by $U(3)$, as can be inferred from Fig. 8.

Octonionic special relativity, complex octonions, and the algebra $\mathbb{R} \times \mathbb{C} \times \mathbb{H} \times \mathbb{O}$

The background space-time is now an octonionic space-time with coordinate vector $\mathbf{x}=$ $x_{0} e_{0}+x_{1} e_{1}+x_{2} e_{2}+x_{4} e_{4}+x_{3} e_{3}+x_{5} e_{5}+x_{6} e_{6}+x_{7} e_{7}$, and the corresponding eight-vector for 
a particle in this octonionic special relativity is $\mathbf{q}_{\mathbf{i}}=q_{0} e_{0}+q_{1} e_{1}+q_{2} e_{2}+q_{4} e_{4}+q_{3} e_{3}+q_{5} e_{5}+$ $q_{6} e_{6}+q_{7} e_{7}$. In ordinary relativity, the $q_{i}$ are real numbers, but now in trace dynamics they are bosonic or fermionic matrices. The space-time symmetry group is the automorphism group $G_{2}$ of the octonions, shown in Fig. 8, along with its maximal sub-groups, which reveal the standard model along with its $4 \mathrm{D}$ Lorentz symmetry. The Lagrangian is the same as in (86) above, but now written on the $8 \mathrm{D}$ octonionic space-time. As a result, $q_{B}$ and $q_{F}$ have component indices $(3,5,6,7)$ whereas their time derivatives have indices $(0,1,2,4)$. This is the Lagrangian analysed in the main part of the present paper and it now includes quarks as well as leptons, along with all twelve standard model gauge bosons plus two Lorentz bosons.

We note the peculiarity that the weak part of the Lorentz-weak symmetry of the leptons, obtained by extending the Lorentz symmetry, intersects with the electr-color sector provided by $U(3) \sim S U(3) \times U(1)$. This strongly suggests that the lepton part of the weak sector can be deduced from the electro-color symmetry. This is confirmed by the earlier work of Stoica [9], Furey [8] and our own earlier work [4].

We see that this Lagrangian is a natural generalisation of Newtonian mechanics and 4D special relativity to the last of the division algebras, the octonions, which represent a 10D Minkowski space-time because of the homomorphism $S L(2, \mathbb{O})=S O(9,1)$.

\section{Emergent quantum field theory}

In the entire discussion above, relating generalised trace dynamics to the standard model, we have made no reference to quantum field theory. The pre-quantum, pre-space-time matrix-valued Lagrangian dynamics which we have constructed above, reveals the standard model and its symmetries (including the Lorentz symmetry) without any fine tuning. Quantum field theory, and classical space-time, are emergent from this pre-theory, after coarse-graining the underlying theory over time-scales much larger than Planck time, in the spirit of Adler's trace dynamics.

String theory is pre-space-time, but not pre-quantum. Trace dynamics is pre-quantum, but not pre-space-time. The octonionic theory [O-theory] is pre-space-time and prequantum. It generalises trace dynamics to a pre-quantum, pre-space-time theory. The O-theory is not intended as an alternative to quantum field theory. Rather, it is applica-

ble in those circumstances when a background classical time is not available for writing down the rules of QFT. Then, the O-theory also reveals itself to be pre-quantum. When a 
background classical time becomes available, O-theory coincides with QFT and is no longer pre-quantum. O-theory reveals the symmetries of the standard model without any finetuning, and also shows a route for determining the free parameters of the standard model. This comes about because the background non-commutative spacetime fixes the properties of the allowed elementary particles. In this way, O-theory has a promising potential to tell us, in a mathematically precise way, where the standard model, and classical space-time, come from. The O-theory is not a Grand Unified Theory [GUTs]. GUTs determine internal symmetries by making specific choices for the internal symmetry group, while classical space-time and QFT are kept intact. In contrast to this, the O-theory retains neither QFT nor a classical space-time. The symmetries of O-theory are a unification of internal and spacetime symmetries, in the spirit of a Kaluza-Klein theory.

The diagram below lists the three main steps in which the octonionic theory is developed. Current investigation is focused at the third step.

The emergence of standard quantum field theory on a classical space-time background is a result of coarse-graining and spontaneous localisation and has been described in our earlier papers $[10,12]$. Spontaneous localisation gives rise to macroscopic classical bodies and 4D classical space-time. From the vantage point of this space-time those STM atoms which have not undergone spontaneous localisation appear, upon coarse-graining of their dynamics, as they are conventionally described by quantum field theory on a 4D classical space-time. Operationally, the transition from the action of the pre-spacetime pre-quantum theory is straightforward to describe. Suppose the relevant term in the action of the pre-theory is denoted as $\int d \tau\left[\operatorname{Tr}\left[T_{1}\right]+\operatorname{Tr}\left[T_{2}\right]+\operatorname{Tr}\left[T_{3}\right]\right]$. Say for instance the three terms respectively describe the electromagnetic field, the action of a $W$ boson on an electron, and the action of a gluon on an up quark. Then, the corresponding action for conventional QFT will be recovered as:

$$
\int d \tau\left[\operatorname{Tr}\left[T_{1}\right]+\operatorname{Tr}\left[T_{2}\right]+\operatorname{Tr}\left[T_{3}\right]\right] \rightarrow \int d \tau \int d^{4} x\left[\left[T_{1 Q F T}\right]+\left[T_{2 Q F T}\right]+\left[T_{3 Q F T}\right]\right]
$$

The trace has been replaced by the space-time volume integral, and each of the three terms have correspondingly been replaced by the conventional field theory actions for the three cases: conventional action for the electromagnetic field, for the $W$ boson acting on the electron, and for the gluon acting on the up quark. In this way, QFT is recovered from the 


\section{Quantum theory without classical time, as a route to quantum gravity and unification}

TRACE DYNAMICS A pre-quantum theory

[A matrix-valued Lagrangian dynamics for fermions and Yang-Mills fields]

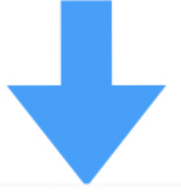

GENERALISED TRACE DYNAMICS

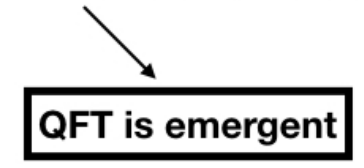

A pre-quantum, pre-spacetime theory

[A matrix-valued Lagrangian dynamics for fermions and Yang-Mills fields and gravitation]
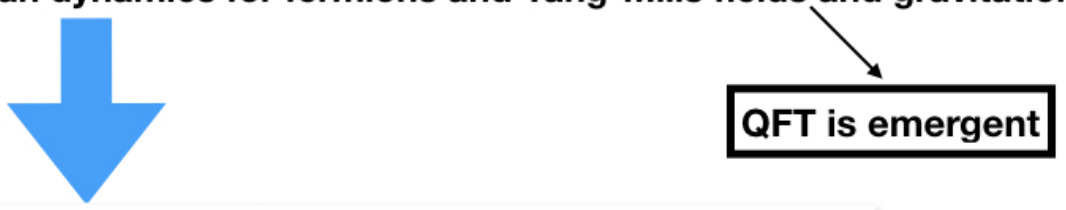

\section{GENERALISED TRACE DYNAMICS on an OCTONIONIC SPACE-TIME}

[Evidence that the theory could possibly describe the standard model, and determine free parameters of the standard model.]

FIG. 11. The pre-space-time, pre-quantum octonionic theory in three key steps. The degrees of freedom are 'atoms of space-time-matter' [STM]. An STM atom is an elementary fermion along with all the fields that it produces. The action for an STM atom resembles a 2-brane in a 10+1 dimensional Minkowski spacetime. The fundamental universe is made of enormously many STM atoms. From here, quantum field theory is emergent upon coarse-graining the underlying fundamental theory.

pre-theory.

However, by starting from the pre-theory, we can answer questions which the standard model cannot answer. We know now why the standard model has the symmetries it does, and why the dimensionless free parameters of the standard model take the values they do. These are fixed by the algebra of the octonions which defines the 8D octonionic spacetime in the pre-theory. While this is work in progress, it provides a promising avenue for understanding the origin of the standard model and its unification with gravitation. 


\section{REFERENCES}

[1] Stephen L. Adler, "Generalized quantum dynamics," Nucl. Phys. B 415, 195 (1994).

[2] Stephen L. Adler and Andrew C. Millard, "Generalised quantum dynamics as pre-quantum mechanics," Nucl. Phys. B 473, 199 (1996).

[3] Stephen L. Adler, Quantum theory as an emergent phenomenon (Cambridge University Press, 2004).

[4] Tejinder P. Singh, "Trace dynamics and division algebras: towards quantum gravity and unification." Zeitschrift für Naturforschung A 76, 131, DOI: https://doi.org/10.1515/zna2020-0255, arXiv:2009.05574v44 [hep-th] (2020).

[5] Tejinder P. Singh, "Quantum theory without classical time: octonions, and a theoretical derivation of the fine structure constant 1/137," https://www.preprints.org/manuscript/202105.0370/v1 (2021).

[6] Cohl Furey, "Standard model physics from an algebra? Ph. D. thesis, university of Waterloo," arXiv:1611.09182 [hep-th] (2015).

[7] Cohl Furey, "Three generations, two unbroken gauge symmetries, and one eight-dimensional algebra," Phys. Lett. B 785, 1984 (2018).

[8] Cohl Furey, "SU(3) $C \times S U(2)_{L} \times U(1)_{Y}\left(\times U(1)_{X}\right)$ as a symmetry of division algebraic ladder operators," Euro. Phys. J. C 78, 375 arXiv:1806.00612 [hep-th] (2018).

[9] Ovidiu Cristinel Stoica, "The standard model algebra (Leptons, quarks and gauge from the complex algebra Cl(6))," Advances in Applied Clifford Algebras 28, 52 arXiv:1702.04336 [hepth] (2018).

[10] Maithresh Palemkota and Tejinder P. Singh, "Proposal for a new quantum theory of gravity III: Equations for quantum gravity, and the origin of spontaneous localisation," Zeitschrift für Naturforschung A 75, 143 (2019 DOI:10.1515/zna-2019-0267 arXiv:1908.04309).

[11] Tejinder P. Singh, "Octonions, trace dynamics and non-commutative geometry: a case for unification in spontaneous quantum gravity," Zeitschrift für Naturforschung A DOI: https://doi.org/10.1515/zna-2020-0196, 1051, arXiv:2006.16274v2 (2020).

[12] Meghraj M S, Abhishek Pandey, and Tejinder P. Singh, "Why does the Kerr-Newman black hole have the same gyromagnetic ratio as the electron?" submitted for publication 
arXiv:2006.05392 (2020).

[13] Geoffrey M. Dixon, Division algebras, octonions, quaternions, complex numbers and the algebraic design of physics (Kluwer, Dordrecht, 1994).

[14] C. H. Tze and F. Gursey, On the role of division, Jordan and related algebras in particle physics (World Scientific Publishing, 1996).

[15] J. Chisholm and R. Farwell, "Clifford geometric algebras: with applications to physics, mathematics and engineering," (Birkhauser, Boston, 1996 Ed. W. R. Baylis) p. 365.

[16] G. Trayling and W. Baylis, "A geometric basis for the standard-model gauge group," J. Phys. A: Math. Theor. 34, 3309 (2001).

[17] Michel Dubois-Violette, "Exceptional quantum geometry and particle physics," Nuclear Physics B 912, 426-449 (2016).

[18] Ivan Todorov, "Exceptional quantum algebra for the standard model of particle physics," Nucl. Phys. B 938, 751 arXiv:1808.08110 [hep-th] (2019).

[19] Michel Dubois-Violette and Ivan Todorov, "Exceptional quantum geometry and particle physics II," Nucl. Phys. B 938, 751-761 arXiv:1808.08110 [hep-th] (2019), arXiv:1808.08110 [hep-th].

[20] Ivan Todorov and Svetla Drenska, "Octonions, exceptional Jordan algebra and the role of the group $F_{4}$ in particle physics," Adv. Appl. Clifford Algebras 28, 82 arXiv:1911.13124 [hep-th] (2018), arXiv:1805.06739 [hep-th].

[21] Ivan Todorov, "Jordan algebra approach to finite quantum geometry," in PoS, Vol. CORFU2019 (2020) p. 163.

[22] Rafal Ablamowicz, "Construction of spinors via Witt decomposition and primitive idempotents: A review," in Clifford algebras and spinor structures, edited by Rafal Ablamowicz and P. Lounesto (Kluwer Acad. Publ., 1995) p. 113.

[23] John C. Baez, "The octonions," Bull.Am.Math.Soc. 39 (2002), arXiv:math/0105155 [math.RA].

[24] John C. Baez, "Division algebras and quantum theory," Foundations of Physics 42, 819-855 (2011).

[25] John C. Baez and John Huerta, "The algebra of grand unified theories," (2009 arXiv:0904.1556 [hep-th]), arXiv:0904.1556 [hep-th].

[26] Carlos Castro Perelman, " $R \times C \times H \times O$ valued gravity as a grand unified field theory," 
Advances in Applied Clifford Algebras 29, 22 (2019).

[27] Adam B. Gillard and Niels G. Gresnigt, "Three fermion generations with two unbroken gauge symmetries from the complex sedenions," The European Physical Journal C 79, 446, arXiv:1904.03186 [hep-th] (2019).

[28] Ichiro Yokota, "Exceptional Lie groups," arXiv:0902.043 [math.DG] (2009).

[29] Tevian Dray and Corinne Manogue, "The exceptional Jordan eigenvalue problem," Int. J. Theo. Phys. 28, 2901 arXiv:math-ph/9910004v2 (1999).

[30] Tevian Dray and Corinne Manogue, "Octonions, $\mathrm{E}_{6}$ and particle physics," J.Phys.Conf.Ser. 254, 012005 arXiv:0911.2253 (2010).

[31] A. Garrett Lisi, "An exceptionally simple theory of everything," arXiv:0711.0770 [hep-th] (2007).

[32] Pierre Ramond, "Introduction to exceptional Lie groups and algebras," https://inspirehep.net/literature/111550 CALT-68-577 (1976).

[33] Ivan Todorov and Michel Dubois-Violette, "Deducing the symmetry of the standard model fom the automorphism and structure groups of the exceptional Jordan algebra," arXiv:1806.09450 [hep-th] (2018).

[34] Adam B. Gillard and Niels Gresnigt, "The $\mathrm{Cl}(8)$ algebra of three fermion generations with spin and full internal symmetries," arXiv:1906.05102 (2019).

[35] A. Adrien Albert, "On a certain algebra of quantum mechanics," Annals of Mathematics 35, 65 (1933).

[36] P. Jordan, John von Neumann, and E. Wigner, "On an algebraic generalisation of the quantum mechanical formalism," Ann. Math. 35, 65 (1933).

[37] Rajrupa Mondal and Vatsalya Vaibhav, "Private communication," (2021).

[38] K. A. Olive; et al. (Particle Data Group) (2014), "Review of particle properties," Chinese Physics C. 38 (9) 38, 1 (2014).

[39] Tejinder P. Singh, "Quantum gravity, minimum length and hologaphy," Pramana - J. Phys. 95, 40, arXiv:1910.06350 (2021).

[40] Roberto Onofrio, "High energy density implications of a gravitoweak unification scenario," Mod. Phy. Letts. A 29, 1350187 (2014).

[41] Latham Boyle, "The standard model, the exceptional Jordan algebra, and triality," e-print , arXiv:2006.16265v1 [hep-th] (2020). 
[42] Vivan Bhatt, Rajrupa Mondal, Vatsalya Vaibhav, and Tejinder P. Singh, "Exceptional Jordan algebra, Majorana neutrinos, and mass-ratios of charged leptons," in preparation (2021).

[43] E. Corrigan and T. J. Hollowood, "Exceptional Jordan algebra and the superstring," Commun. Math. Phys. 122, 393 (1989).

[44] P. Goddard, W. Nahm, D. Olive, H. Ruegg, and A. Schwimmer, "Fermions and octonions," Commun. Math. Phys. 112, 385 (1987).

[45] Shinichi Deguchi, Yuki Fujiwara, and Kunihiko Nakano, "Two quantization approaches to the Bateman oscillator model," Ann. Physics 403, 34 arXiv:1807.04403 [quant-ph] (2019).

[46] Cohl Furey and Mia Hughes, in preparation, 2021. 